\title{
A geometric approach to error estimates for conservation laws posed on a spacetime
}

\author{
Paulo Amorim ${ }^{1}$, Philippe G. LeFloch ${ }^{2}$, and Wladimir Neves ${ }^{3}$
}

\begin{abstract}
We consider a hyperbolic conservation law posed on an $(N+1)$-dimensional spacetime, whose flux is a field of differential forms of degree N. Generalizing the classical Kuznetsov's method, we derive an $L^{1}$ error estimate which applies to a large class of approximate solutions. In particular, we apply our main theorem and deal with two entropy solutions associated with distinct flux fields, as well as with an entropy solution and an approximate solution. Our framework encompasses, for instance, equations posed on a globally hyperbolic Lorentzian manifold.
\end{abstract}

\section{Introduction}

This paper provides a general framework leading to error estimates for hyperbolic conservation laws posed on an $(N+1)$-dimensional manifold $M$, referred to as a spacetime and, in particular, leading to a sharp estimate for the difference, measured in the $L^{1}$ norm, between an exact solution and an approximate solution. The present paper can be regarded as a generalization to manifolds of a contribution by Bouchut and Perthame [4], who recast in a concise form the pioneering works of Kruzkov and Kuznetsov [6, 7, 8] for hyperbolic conservation laws posed on the flat (Euclidian) spacetime. We are thus interested here in extending these results to conservation laws defined on manifolds, and develop a physically more realistic setting when geometrical effects are now taken into account.

Motivated by the case of the shallow water equations on the sphere, the theory of hyperbolic conservation laws on manifolds has been developed in recent years by LeFloch together with collaborators. In particular, well-posedness results have been obtained in [3, 9, 11], and convergence results for finite volume schemes in [1, 2], while an error estimate in the case of a Riemannian manifold was derived in [10]. For further results on the well-posedness theory, we also refer the reader to contributions by Panov in [12,13] and, on the finite volume schemes, to the earlier work [5].

Recently, in [11], LeFloch and Okutmustur introduced a framework based on differential forms and dealt with conservation laws defined on an $(N+1)$-dimensional manifold $M$. In their formulation, the flux of the equation is given by a field of $N$-forms, rather than by a vector field as was the case in earlier works. The formulation based on $N$-forms is geometrically natural in that only minimal assumptions on the geometrical structure are required in order to establish the well-posedness of the initial value problem.

\footnotetext{
${ }^{1}$ Centro de Matemática e Aplicações Fundamentais, Departamento de Matemática, Universidade de Lisboa, Av. Prof. Gama Pinto 2, 1649-003 Lisboa, Portugal. E-mail: pAmorim@ptmat.fc.ul.pt

${ }^{2}$ Laboratoire Jacques-Louis Lions \& Centre National de la Recherche Scientifique, Université Pierre et Marie Curie (Paris 6), 4 Place Jussieu, 75252 Paris, France. E-mail: pgLeFloch@gmail.com

${ }^{3}$ Instituto de Matemática, Universidade Federal do Rio de Janeiro, C.P. 68530, Cidade Universitária 21945-970, Rio de Janeiro, Brazil. E-mail: Wladimir@im.ufrj.br

AMS Subject Classification. Primary: 35L65. Secondary: 58J45. Key words and phrases. Hyperbolic conservation law, flux field, spacetime, differential form, entropy solution, error estimate. Completed in July 2010. Revised in April 2011. To appear in: Nonlinear Analysis (2011).
} 
Considering hyperbolic conservation laws posed on a spacetime, we derive here a coordinatefree error estimate in the $L^{1}$-norm, which involves, on one hand, an exact weak solution satisfying all entropy inequalities and, on the other hand, an approximate solution for which the entropy dissipation measures contain some (positive) "error terms". Our assumptions on the manifold are minimal and, in particular, no metric is needed and no volume form is a priori prescribed. Indeed, our method only requires a suitable "hyperbolicity condition", and the prescription of a certain family of mollifiers (similar to mollifiers that may be determined from the distance function when a Riemann metric is given on the manifold). Still, we observe below that, to the flux-field defining the conservation law, one can associate a natural choice of volume form defined on the manifold. In addition, from our main theorem we deduce, on one hand, the $L^{1}$-contraction property of the semigroup of entropy solutions and, on the other hand, a sharp estimate comparing together the solutions of two conservation laws with different flux fields.

In the second part of this paper, we investigate the case of a conservation law defined on a Lorentzian manifold. Here, the presence of a metric allows us to refine our estimates. For instance, a suitable family of mollifiers is provided by the metric structure of the manifold, and the error terms can be given in a more explicit way, since they are naturally written using the metric. More importantly, the introduction of a metric allows us to consider second-order error terms in the approximate solutions. This allows us to apply our main result further, and derive error estimates for a nonlinear diffusion model.

We may summarize the main difficulties overcome in this paper as follows. First of all, since the conservation law under consideration is posed on a non-flat manifold, the geometry of that manifold must be taken into account in, for instance, the formulation of approximate schemes and the analysis of their convergence. It has been pointed out that geometric effects occur which change the qualitative properties of solutions [1]. Spacetimes and, in particular, Lorentzian manifolds may not be "invariant by translation" in the time direction, and the time and spatial geometries are intertwined, giving rise to phenomena not present in the Euclidian or Riemannian set-up. The main difficulty dealt with here lies in the lack of geometric structure on the spacetime which makes it difficult to deploy the analytical techniques used when a metric is prescribed. To circumvent this problem, we assume the existence of a suitable family of mollifiers, which are adapted to the sole structure available on the manifold, namely the family of flux fields of the conservation law. For this reason, our estimate in Theorem 2.4 is, later on in Theorem 3.2 specialized to the case that a metric is specified. (See also an earlier result in [10].) Importantly, all of the estimates established in the present paper are coordinate-free.

An outline of the paper follows. Section 2 is devoted to the general framework. First of all, in Section 2.1, we recall from [11] some basic concepts about conservation laws posed on a spacetime. We then introduce the notion of admissible mollifiers, which allows us to state our main result, in Theorem 2.4 below. Then, in Section 2.4 we discuss our first application concerning two conservation laws with distinct flux fields. The rest of the section is devoted to the proof of Theorem 2.4. In the second part of the paper encompassing the whole of Section 3 . we treat the special case of conservation laws posed on a Lorentzian manifold, and state our error estimate in Theorem 3.2. We are then in a position to provide two more applications in Section 3.3 and 3.4. Section 3.5 contains a proof of Theorem 3.2

\section{Error estimates for a spacetime}

\subsection{Conservation laws based on differential forms}

Let $M$ be an $(N+1)$-dimensional manifold (with smooth topological structure), which we refer to as a spacetime. Denote by $\Lambda^{k}(M)$ the space of all smooth fields of $k$-differential forms on $M$, and by $d: \Lambda^{k}(M) \rightarrow \Lambda^{k+1}(M)$ the exterior derivative operator.

Following LeFloch and Okutmustur [11], we recall the formulation of the initial value problem for hyperbolic conservation laws posed on a spacetime. The setting is based on differential forms 
and the conservation law reads

$$
d(\omega(u))=0 \quad \text { on } \quad M,
$$

where $u: M \rightarrow \mathbb{R}$ is the unknown function and the given family of smooth differential $N$-forms $\omega=\omega(u) \in \Lambda^{N}(M)$ depends smoothly upon the variable $u$ and is referred to as the flux field of the conservation law. The field $\omega$ is said to be geometry compatible if it is exact, that is,

$$
(d \omega)(\bar{u})=0, \quad \bar{u} \in \mathbb{R} .
$$

Moreover, (2.1) is supplemented with the initial condition

$$
\left.u\right|_{\mathcal{H}_{0}}=u_{0},
$$

where $u_{0}$ is a given data defined on a hypersurface $\mathcal{H}_{0} \subset M$. Throughout this paper we assume that the data $u_{0}$ and, therefore, the unknown function (thanks to the maximum principle) is bounded, that is,

$$
-C_{0} \leq u_{0} \leq C_{0},
$$

for some $C_{0}>0$. We tacitly restrict all values of $u$ to lie in the interval $\left[-C_{0}, C_{0}\right]$, and we point out that all of our estimates in this paper depend implicitly on this constant $C_{0}$.

Following [11], we introduce the following notion of global hyperbolicity: the manifold $M$ is foliated by hypersurfaces,

$$
M=\bigcup_{t \geq 0} \mathcal{H}_{t}
$$

for some (smooth) time-function $t$, where each slice $\mathcal{H}_{t}$ has the same topology as $\mathcal{H}_{0}$ which we assume to be a (smooth), compact, orientable $N$-manifold. For each $T>0$, we use the notation

$$
M_{T}:=\bigcup_{0 \leq t \leq T} \mathcal{H}_{t} .
$$

Moreover, given any hypersurface $\mathcal{H}$, we denote by $i: \mathcal{H} \rightarrow M$ the canonical imbedding and by $i^{*}$ its pullback, taking forms in $\Lambda^{k}(M)$ to forms in $\Lambda^{k}(\mathcal{H})$.

Definition 2.1. Let $M$ be a foliated manifold (2.4) and $\omega=\omega(\bar{u})$ be a flux field. The conservation law (2.1) is said to satisfy the global hyperbolicity condition if for all $t \geq 0$ the $N$-form field $i_{\mathcal{H}_{t}}^{*} \omega(0)$ is a volume form on $\mathcal{H}_{t}$, and there exist constants $0<\underline{c}<\bar{c}$ independent of t such that for all $\bar{u} \in\left[-C_{0}, C_{0}\right]$

$$
\underline{c} i_{\mathcal{H}_{t}}^{*} \partial_{u} \omega(0) \leq i_{\mathcal{H}_{t}}^{*} \partial_{u} \omega(\bar{u}) \leq \bar{c} i_{\mathcal{H}_{t}}^{*} \partial_{u} \omega(0),
$$

as inequalities between $N$-forms defined on the slice $\mathcal{H}_{t}$.

In particular, the condition (2.5) implies that for each non-empty smooth hypersurface $e \subset \mathcal{H}_{t}$ the integral

$$
\int_{e} i_{e}^{*} \partial_{u} \omega(\bar{u})
$$

is positive, and its ratio with $\int_{e} i_{e}^{*} \partial_{u} \omega(0)$ is bounded above and below.

Recall also the following definition of entropy solutions.

Definition 2.2. A smooth field of $N$-forms $\Omega=\Omega(\bar{u})$ is called a (convex) entropy flux field for the conservation law (2.1) if there exists a (convex) function $U: \mathbb{R} \rightarrow \mathbb{R}$ such that

$$
\Omega(\bar{u})=\int_{0}^{\bar{u}} \partial_{u} U(v) \partial_{u} \omega(v) d v, \quad \bar{u} \in \mathbb{R} .
$$

A measurable and bounded function $u: M \rightarrow \mathbb{R}$ is called an entropy solution to the Cauchy problem (2.1)-(2.2) if for every (compactly supported and smooth) non-negative test-function $\varphi$, the following entropy inequalities hold for any convex entropy pair $(U, \Omega)$

$$
\int_{M}\left(d \varphi \wedge \Omega(u)+\varphi(d \Omega)(u)-\varphi \partial_{u} U(u)(d \omega)(u)\right)+\left.\int_{\mathcal{H}_{0}} \varphi\right|_{\mathcal{H}_{0}} i_{\mathcal{H}_{0}}^{*} \Omega\left(u_{0}\right) \geq 0 .
$$


Under the global hyperbolicity condition above, the flux of the equation naturally induces an $((N+1)$-dimensional) reference volume form on $M$, namely

$$
\bar{\alpha}:=d t \wedge \partial_{u} \omega(0) .
$$

We emphasize that this volume form depends on, both, the flux field (at the state $\bar{u}=0$ ) and the chosen foliation. On the other hand, a more fundamental structure on $M$ is provided by the family of $N$-form flux $\omega=\omega(\bar{u})$ which determines the conservation law under consideration.

Finally, we recall from [11] that, under the global hyperbolicity condition, the initial value problem (2.1)-(2.2) admits a unique entropy solution that depends Lipschitz continuously in the $L^{1}$ norm upon its initial data.

\subsection{Approximate solutions}

The main objective of this paper is to provide a general framework for the derivation of error estimates for hyperbolic conservation laws defined on a spacetime. In our statements and proofs we will use the Kruzkov entropies

$$
U(u, k)=|u-k|, \quad k \in \mathbb{R}
$$

with entropy flux

$$
\Omega(u, k)=\operatorname{sgn}(u-k)(\omega(u)-\omega(k)) .
$$

Let $v$ be an (exact) entropy solution to the conservation law (2.6), satisfying therefore

$$
d(\Omega(v, l))+G(v, l) \leq 0, \quad l \in \mathbb{R}
$$

in the sense of distributions, where $G=G(v, l)$ are $(N+1)$-forms defined by

$$
G(v, l):=\operatorname{sgn}(v-l)(d \omega)(l) \text {. }
$$

Following now Bouchut and Perthame [4] and in order to deal with approximate solutions, it is convenient to introduce Radon measures to estimate error terms that arise in an approximate version of the entropy inequalities. Furthermore, in our setting, rather than functions or vector fields, we have to deal with $N$-form fields on the manifold $M$. Hence, we now introduce Radon measure-valued fields of $N$-forms. For instance, a distributional $(N+1)$-form is an element of the dual of the space of test-functions on $M$, while a (scalar) distribution is an element of the dual of the space of (smooth) compactly supported $(N+1)$-forms. In what follows, to keep the notation simple we write, for instance,

$$
\int_{M} \varphi \rho
$$

for the duality bracket between a Radon-measure $(N+1)$-form field $\rho$ and a (continuous) testfunction $\varphi$.

We are now in a position to write the approximate entropy inequalities satisfied by some approximate solution $u: M \rightarrow \mathbb{R}$, that is,

$$
d(\Omega(u, k))+G(u, k) \leq d H_{k}+K_{k}=: E_{k}, \quad k \in \mathbb{R},
$$

where $H_{k}$ is a family of locally Radon measure-valued $N$-form fields and $K_{k}$ a family of locally Radon measure-valued $(N+1)$-form fields. We make the following key assumption on these error terms: there exist a non-negative Radon measure-valued $N$-form field $\alpha_{H}$ and a non-negative Radon measure-valued $(N+1)$-form field $\alpha_{K}$ such that for every 1 -form test-field $\gamma$ and every test-function $\varphi \geq 0$

$$
\begin{aligned}
& \sup _{k \in \mathbb{R}}\left|\int_{M} H_{k} \wedge \gamma\right| \leq\left|\int_{M} \alpha_{H} \wedge \gamma\right|, \\
& \sup _{k \in \mathbb{R}} \int_{M} \varphi K_{k} \leq \int_{M} \varphi \alpha_{K} .
\end{aligned}
$$


Next, we need to introduce a suitable generalization of Kruzkov and Kuznetsov's mollifiers. This is straightforward on a Riemannian manifold, by using the canonical distance function, but in the present formalism, we need the following new notion.

In what follows, if $\zeta=\zeta(p, q)=\zeta_{p, q}$ is a function on $M \times M$, then $d_{p} \zeta$ and $d_{q} \zeta$ denote its differentials with respect to the first and second arguments, respectively. We use the notation $\zeta_{p}$ for the function $p \mapsto \zeta(p, q)$, for $q$ fixed, and we often specify the integration variable in each integral to avoid confusion, by adding a subscript to the volume form under consideration. To a sequence of functions $\zeta^{\delta}$ we associate their supports $E_{p}^{\delta}:=\operatorname{supp}_{q} \zeta_{q}^{\delta}$. Recalling that $\bar{\alpha}$ denotes the volume form (2.7), we also write $|E|:=\int_{E} \bar{\alpha}$ for the volume of a set $E$ and, therefore, $\left|E_{p}^{\delta}\right|=\int_{E_{p}^{\delta}} \bar{\alpha}_{q}$. We also use the notation $f_{E} \bar{\alpha}:=|E|^{-1} \int_{E} \bar{\alpha}$.

Definition 2.3. Fix a non-negative constant $A$ and a smooth 1-form field $\beta$ defined on $M$. A family of non-negative (compactly supported and smooth) test-functions $\left(\zeta^{\delta}\right)_{\delta>0}$ defined on $M \times M$ is called an $(A, \beta)$-admissible family of mollifiers if the following conditions are satisfied:

1. Unit mass condition: $\int_{M} \zeta_{p, q}^{\delta} \bar{\alpha}_{q}=1$ for each $p \in M$.

2. Sup-norm condition: $\sup _{q \in M} \zeta_{p, q}^{\delta} \leq\left|E_{p}^{\delta}\right|^{-1}$ for each $p \in M$.

3. Differential condition:

$$
\iint_{M \times M}\left|d_{p} \zeta_{p, q}^{\delta} \wedge \gamma_{p, q}\right| \leq \frac{1}{\delta} \int_{M} \oint_{E_{p}^{\delta}}\left|\beta_{p} \wedge \gamma_{p, q}\right|
$$

for each test $(2 N+1)$-form field $\gamma$.

4. Symmetry condition: for each $\bar{u} \in\left[-C_{0}, C_{0}\right]$ and with $\gamma:=\partial_{u} \omega(\bar{u})$

$$
\begin{aligned}
& \iint_{M \times M} \varphi_{p, q}\left(d_{p} \zeta_{p, q}^{\delta} \wedge \gamma_{p} \wedge \bar{\alpha}_{q}+d_{q} \zeta_{p, q}^{\delta} \wedge \gamma_{q} \wedge \bar{\alpha}_{p}\right) \\
& \leq A \int_{M} \oint_{E_{p}^{\delta}} \varphi_{p, q} \bar{\alpha}_{p} \wedge \bar{\alpha}_{q}
\end{aligned}
$$

for every bounded function $\varphi: M \times M \rightarrow \mathbb{R}^{+}$.

On a Riemannian manifold the distance function allows one to define such a family of testfunctions. In general, the test-functions should be defined in each application by taking advantage of special properties of the given family of approximate solutions (cf. examples below).

These assumptions arise from natural requirements on the supports $E_{p}^{\delta}$, volumes $\left|E_{p}^{\delta}\right|$, and testfunctions $\zeta^{\delta}$. They take the proposed form, due to the lack of metric structure on the manifold. For instance, Condition (2) is a "smallness" condition on the mollifiers, while Condition (1) replaces the unit integral property of standard mollifiers in Euclidian or Riemannian manifolds.

Condition (4) is a symmetry property, enjoyed in the Euclidian space by $\zeta^{\delta}(p, q)=\eta(\ell(p, q))$, which takes then the much simpler form $d_{p} \zeta^{\delta}=-d_{q} \zeta^{\delta}$, when $\ell(p, q)$ denotes the Euclidian distance function. In a Riemannian or Lorentzian setting, a similar property holds, but one needs to use parallel transport to compare $d_{p} \zeta$ and $d_{q} \zeta$ (see the proof of Theorem 3.2 below). Our condition above is intended to encompass all situations, when no metric is naturally available on the manifold. The inequality in Condition (4) in Definition 2.3 is motivated by the following formal calculation, in wich $M=\mathbb{R}$ and $\zeta$ coincides with a function $\eta$ composed with the distance function $\ell$ :

$$
\begin{aligned}
d_{p} \zeta \gamma(p)+d_{q} \zeta \gamma(q) & =d_{p} \zeta(\gamma(p)-\gamma(q)) \\
& \leq\left|\eta^{\prime}(\ell(p, q))\right| \sup _{\mathbb{R}}\left|\gamma^{\prime}\right| \ell(p, q) .
\end{aligned}
$$

Now, $\eta$ will usually be a standard mollifier, and thus in this example, $\left|\eta^{\prime}\right| \leq \delta^{-2} \lesssim(\delta|\operatorname{supp} \eta|)^{-1}$. Since $\ell(p, q) \leq \delta$, the expression above is bounded by $|\operatorname{supp} \eta|^{-1} \sup _{\mathbb{R}}\left|\gamma^{\prime}\right|$, and Condition (4) is a 
generalization of this formal argument, in an integral form. This is also a condition on the size of the support of $\zeta^{\delta}$, since it somehow generalizes the fact that in Riemannian space, $\ell(p, q) \leq \delta$ if $q$ is in the support of $\zeta_{p}^{\delta}$ and if those supports are geodesic balls of diameter $\delta$. This assumption is necessary since, without a metric on the manifold, it seems difficult to reproduce the above argument in a geometric (i.e., coordinate independent) way.

Note, however, that if one is interested in some particular problem, it is not hard to express the constant $A$ in Condition (4) as a (possibly coordinate dependent) quantity involving derivatives of $\gamma$.

As for Condition (3) it amounts to a uniform upper bound on the form fields $d_{p} \zeta$ by a certain 1 -form field $\beta$. It is analogous to the bound $\left|\nabla \zeta^{\delta}\right| \leq C \delta^{-N-2}$ enjoyed by the standard molifiers in the Euclidian and Riemannian cases [4, 10].

\subsection{Statement of the error estimate}

Our main result in the present paper is now stated. Recall that all values $u$ under consideration belong to the interval $\left[-C_{0}, C_{0}\right]$.

Theorem 2.4 (Error estimate for conservation laws on a spacetime). Consider the conservation law (2.1) with flux field $\omega$, posed on a spacetime $M$ satisfying the foliated condition (2.4) and the global hyperbolicity condition (2.5) for some $\underline{c}, \bar{c}$. Let $\zeta^{\delta}$ be an $(A, \beta)$-admissible family of mollifiers associated with some non-negative constant $A$ and 1 -form field $\beta$. Consider two functions $u(t), v(t): \mathcal{H}_{t} \rightarrow \mathbb{R}$ that belong to $L^{1}\left(\mathcal{H}_{t}\right)$ for each $t \geq 0$ and are right-continuous in $t$. Assume moreover that $v$ is an (exact) entropy solution to the conservation law (2.8), and that $u$ satisfies the approximate entropy inequalities (2.9) for some $H_{k}, K_{k}, \alpha_{H}, \alpha_{K}$ satisfying the bounds (2.10) and such that $i_{\mathcal{H}_{t}}^{*} \alpha_{H}$ belongs to $L^{1}\left(\mathcal{H}_{t}\right)$ for all $t$. Then, the following $L^{1}$-type estimate holds for all $\delta>0$ and $T>0$

$$
\int_{\mathcal{H}_{T}} i^{*} \Omega(u, v) \leq \int_{\mathcal{H}_{0}} i^{*} \Omega(u, v)+R^{\delta}[v]+R^{\delta}[\omega]+R^{\delta}[\alpha],
$$

where

$$
\begin{gathered}
R^{\delta}[v]:=\sup _{t \in(0, T)} \int_{\mathcal{H}_{t}} i * \bar{\alpha}_{p} \oint_{E_{p}^{\delta}}\left|v_{p}-v_{q}\right| B_{q}, \\
R^{\delta}[\omega]:=\int_{M_{T}} \oint_{E_{p}^{\delta}}\left|d \omega_{p}\left(v_{q}\right) \wedge \bar{\alpha}_{q}-d \omega_{q}\left(v_{q}\right) \wedge \bar{\alpha}_{p}\right|, \\
R^{\delta}[\alpha]:=\frac{1}{\delta} \int_{M_{T}}\left|\beta \wedge \alpha_{H}\right|+\int_{\mathcal{H}_{0} \cup \mathcal{H}_{T}}\left|i^{*} \alpha_{H}\right|+\int_{M_{T}} \alpha_{K},
\end{gathered}
$$

and $B$ is an $(N+1)$-form field in $q$ defined by

$$
B_{q}:=(2 \bar{c}+T A) \bar{\alpha}_{q}+T \sup _{u}\left|\partial_{u} d \omega_{q}(u)\right| .
$$

A few remarks about the above theorem are in order. First of all, the terms $R^{\delta}[v]$ and $R^{\delta}[\omega]$ are expected to tend to zero with $\delta$. For instance, when a metric is prescribed on the manifold, the term $R^{\delta}[v]$ is estimated (see Lemma 3.4, below) like in the classical Euclidian case [4]: $R^{\delta}[v] \leq$ $C T \delta \operatorname{TV}(v(0))$, provided $v$ has bounded variation.

Second, under the regularity assumptions on the flux $\omega$, the term $R^{\delta}[\omega]$ is expected to be of order $\mathcal{O}(\delta)$. However, to establish this property, one needs to control the "size" of the sets $E_{p}^{\delta}$, but this cannot be formulated without a notion of distance on $M$. In contrast, Theorem 3.2 below will specialize to the case of a metric on $M$ and on conservation laws based on vector fields, and we will see explicitly that $R^{\delta}[\omega]$ vanishes with $\delta$.

Finally, note that the quantity $\int_{\mathcal{H}_{T}} i^{\star} \Omega(u, v)$ can be seen as a measure of the $L^{1}$-norm of the difference between $u$ and $v$. Indeed, in the Euclidian and Riemannian cases, it reduces to $\int_{\mathcal{H}_{T}} \mid u_{p}-$ $v_{p} \mid d \operatorname{Vol}\left(\mathcal{H}_{t}\right)$. 
Before discussing some applications of the above theorem, it is interesting to consider the special case where the flux field $\omega$ is "geometry compatible".

Corollary 2.5. In addition to the assumptions in Theorem 2.4 assume that the flux field $\omega$ is geometry compatible, in the sense that $(d \omega)(\bar{u})=0$ for each $\bar{u} \in \mathbb{R}$. Then, the following error estimate

$$
\int_{\mathcal{H}_{T}} i^{*} \Omega(u, v) \leq \int_{\mathcal{H}_{0}} i^{*} \Omega(u, v)+R^{\delta}[v]+R^{\delta}[\alpha],
$$

holds for all $T \geq 0$, with $R^{\delta}[v]$ and $R^{\delta}[\alpha]$ defined as in Theorem 2.4

Furthermore, if $v$ is sufficiently smooth so that $R^{\delta}[v] \rightarrow 0$ as $\delta \rightarrow 0$ and if $u$ is also an exact entropy solution, then the $L^{1}$-like distance between $u$ and $v$

$$
t \mapsto \int_{\mathcal{H}_{t}} i^{*} \Omega(u, v)
$$

is non-increasing in time.

The second statement in the above corollary is nothing but the contraction property of the semi-group of entropy solutions.

\subsection{Application (I). Comparing two conservation laws}

Theorem 2.4 applies to conservation laws with "modified" flux, and allows us to estimate the difference between entropy solutions to two distinct conservation laws. Let $\omega$ and $\widetilde{\omega}$ be two geometry-compatible flux fields, and introduce their corresponding Kruzkov entropy flux field

$$
\Omega(v, k)=\operatorname{sgn}(v-k)(\omega(v)-\omega(k)), \quad \widetilde{\Omega}(u, k)=\operatorname{sgn}(u-k)(\widetilde{\omega}(u)-\widetilde{\omega}(k)) .
$$

The solutions $u, v$ under consideration satisfy the entropy inequalities

$$
d(\Omega(v, k)) \leq 0, \quad d(\widetilde{\Omega}(u, k)) \leq 0, \quad k \in \mathbb{R} .
$$

In order to avoid unnecessary technicalities, we may assume that the chain rule applies to expressions involving the functions $u$ and $v$ (i.e. bounded functions with bounded variation, for instance).

Theorem 2.6. Let $u, v$ be to entropy solutions satisfying (2.13) for two flux fields $\omega$ and $\widetilde{\omega}$, and assume that the conditions in Theorem 2.4 hold for both conservation laws. Then, the following two estimates hold:

1. If $v$ is sufficiently regular so that $R^{\delta}[v] \rightarrow 0$ as $\delta \rightarrow 0$, then

$$
\int_{\mathcal{H}_{T}} i^{*} \Omega(u, v) \leq \int_{\mathcal{H}_{0}} i^{*} \Omega(u, v)+C \int_{M_{T}}\left|\partial_{u}(\omega-\widetilde{\omega}) \wedge d v\right|
$$

for some uniform constant $C>0$.

2. If, moreover, $R^{\delta}[v] \leq \delta \bar{R}[v]$, for some constant $\bar{R}[v]$ independent of $\delta$, then

$$
\begin{aligned}
\int_{\mathcal{H}_{T}} i^{*} \Omega(u, v) \leq & \int_{\mathcal{H}_{0}} i^{*} \Omega(u, v)+C\left(\bar{R}[v] \int_{M_{T}}|\beta \wedge Q(\omega, \widetilde{\omega})||u(p)|\right)^{1 / 2} \\
& +\int_{\mathcal{H}_{0} \cup \mathcal{H}_{T}} i^{*} Q(\omega, \widetilde{\omega})|u(p)|
\end{aligned}
$$

with

$$
Q(\omega, \widetilde{\omega})=\sup _{u \neq 0}|\omega(u)-\widetilde{\omega}(u)| /|u|
$$


Proof. In order to apply Theorem 2.4, or more precisely its Corollary 2.5, we need to identify the structure of the relevant approximate conservation laws. To this end, we write

$$
d(\Omega(u, k)) \leq d(\operatorname{sgn}(u-k)((\omega-\widetilde{\omega})(u)-(\omega-\widetilde{\omega})(k)))=: d\left(\gamma_{k}(u)\right) .
$$

To show the estimate (2.14), we set $K_{k}:=d\left(\gamma_{k}(u)\right)$. Using a weak form of the chain rule, we see that

$$
\left|K_{k}\right| \leq\left|\partial_{u}(\omega-\widetilde{\omega}) \wedge d u\right|
$$

Hence, we arrive at the desired estimate (2.14), when $\delta \rightarrow 0$ and after changing the role of $u$ and v.

Second, to establish (2.15), we set $H_{k}:=\gamma_{k}(u)$. Given an arbitrary 1-form field and following arguments in [4] (say that $\omega(0)=\widetilde{\omega}(0)=0$ for simplicity), we obtain

$$
\left|H_{k} \wedge \gamma\right| \leq C\left|\alpha_{H} \wedge \gamma\right|
$$

with $\alpha_{H}:=Q(\omega, \widetilde{\omega})|u|$. Therefore, the estimate (2.15) now follows from Corollary 2.5] by minimizing over the parameter $\delta$. This completes the proof of Theorem 2.6

\subsection{Derivation of the error estimate}

Proof of Theorem 2.4 Let $u$ satisfy the approximate entropy inequalities (2.9), and let $v$ satisfy the (exact) entropy inequalities (2.8). Let $\varphi=\varphi_{p, q}$ be a smooth, compactly supported function on $M \times M$. According to (2.6) and (2.10), for each $k \in \mathbb{R}$ and $q \in M$ we have for $\varphi \geq 0$

$$
\begin{aligned}
& -\int_{M} d_{p} \varphi \wedge \Omega_{p}\left(u_{p}, k\right)+\int_{M} \varphi G_{p}\left(u_{p}, k\right) \\
& \leq \int_{M} \varphi E_{k}=-\int_{M} H_{k} \wedge d_{p} \varphi+\int_{M} K_{k} \varphi \\
& \leq \int_{M}\left|\alpha_{H} \wedge d_{p} \varphi\right|+\int_{M} \alpha_{K} \varphi,
\end{aligned}
$$

thus by taking $k=v_{q}$ we find

$$
\begin{aligned}
& -\int_{M} d_{p} \varphi \wedge \Omega_{p}\left(u_{p}, v_{q}\right)+\int_{M} \varphi G_{p}\left(u_{p}, v_{q}\right) \\
& \leq \int_{M}\left|\alpha_{H} \wedge d_{p} \varphi\right|+\int_{M} \alpha_{K} \varphi .
\end{aligned}
$$

On the other hand, taking $l=u_{p}$ in $(2.8)$ gives

$$
-\int_{M} d_{q} \varphi \wedge \Omega_{q}\left(u_{p}, v_{q}\right)+\int_{M} \varphi G_{q}\left(v_{q}, u_{p}\right) \leq 0 .
$$

Since $q$ is the integration variable, the integrals in (2.17) may be viewed as real-valued functions of $p$. Therefore, we may integrate this function on the manifold, provided a volume form is used. Likewise, we may integrate the inequality (2.16) in $q$. Choosing the form $\bar{\alpha}$ from (2.7), we obtain

$$
\begin{aligned}
& -\int_{M} \bar{\alpha}_{q} \int_{M} d_{p} \varphi \wedge \Omega_{p}\left(u_{p}, v_{q}\right)+\int_{M} \bar{\alpha}_{q} \int_{M} \varphi G_{p}\left(u_{p}, v_{q}\right) \\
& \leq \int_{M} \bar{\alpha}_{q} \int_{M}\left|\alpha_{H} \wedge d_{p} \varphi\right|
\end{aligned}
$$

and

$$
-\int_{M} \bar{\alpha}_{p} \int_{M} d_{q} \varphi \wedge \Omega_{q}\left(u_{p}, v_{q}\right)+\int_{M} \bar{\alpha}_{p} \int_{M} \varphi G_{q}\left(v_{q}, u_{p}\right) \leq 0
$$


Summing up the above two inequalities and applying Fubini's theorem, we obtain

$$
\begin{aligned}
& -\iint_{M \times M} d_{p} \varphi \wedge \Omega_{p}\left(u_{p}, v_{q}\right) \wedge \bar{\alpha}_{q}+d_{q} \varphi \wedge \Omega_{q}\left(u_{p}, v_{q}\right) \wedge \bar{\alpha}_{p} \\
& +\iint_{M \times M} \varphi\left(G_{p}\left(u_{p}, v_{q}\right) \wedge \bar{\alpha}_{q}+G_{q}\left(v_{q}, u_{p}\right) \wedge \bar{\alpha}_{p}\right) \\
& \leq \iint_{M \times M}\left|\alpha_{H} \wedge d_{p} \varphi\right| \wedge \bar{\alpha}_{q}+\alpha_{K} \wedge \bar{\alpha}_{q} \varphi .
\end{aligned}
$$

Let $\left(\zeta^{\delta}\right)_{\delta}$ be an admissible family of mollifiers as in Definition 2.3, and let $\chi_{p}$ be a smooth, compactly supported real function on $M$ to be specified later. We choose the test-functions

$$
\varphi_{p, q}=\chi_{p} \zeta_{p, q}^{\delta}
$$

which leads us to

$$
d_{p} \varphi=\zeta^{\delta} d \chi+\chi d_{p} \zeta^{\delta}, \quad d_{q} \varphi=\chi d_{q} \zeta^{\delta},
$$

and so the inequality (2.18) becomes

$$
\begin{aligned}
& -\iint_{M \times M} \zeta^{\delta} d \chi \wedge \Omega_{p}\left(u_{p}, v_{q}\right) \wedge \bar{\alpha}_{q} \\
& -\iint_{M \times M} \chi\left(d_{p} \zeta^{\delta} \wedge \Omega_{p}\left(u_{p}, v_{q}\right) \wedge \bar{\alpha}_{q}+d_{q} \zeta^{\delta} \wedge \Omega_{q}\left(u_{p}, v_{q}\right) \wedge \bar{\alpha}_{p}\right) \\
& +\iint_{M \times M} \chi \zeta^{\delta}\left(G_{p}\left(u_{p}, v_{q}\right) \wedge \bar{\alpha}_{q}+G_{q}\left(v_{q}, u_{p}\right) \wedge \bar{\alpha}_{p}\right) \\
& \leq \iint_{M \times M}\left|\alpha_{H} \wedge d_{p}\left(\chi \zeta^{\delta}\right)\right| \wedge \bar{\alpha}_{q}+\alpha_{K} \wedge \bar{\alpha}_{q} \chi \zeta^{\delta},
\end{aligned}
$$

which, with obvious notation, has the form

$$
I_{1}-I_{2}+I_{3} \leq I_{4}
$$

\section{The terms $I_{2}$ and $I_{3}$.}

We have

$$
\begin{aligned}
I_{2}:= & \iint_{M \times M} \chi_{p}\left(d_{p} \zeta^{\delta} \wedge \Omega_{p}\left(u_{p}, v_{q}\right) \wedge \bar{\alpha}_{q}+d_{q} \zeta^{\delta} \wedge \Omega_{q}\left(u_{p}, v_{q}\right) \wedge \bar{\alpha}_{p}\right) \\
= & \iint_{M \times M} \chi_{p} d_{q} \zeta^{\delta} \wedge \Omega_{q}\left(u_{p}, v_{p}\right) \wedge \bar{\alpha}_{p} \\
& +\iint_{M \times M} \chi_{p} d_{q} \zeta^{\delta} \wedge\left(\Omega_{q}\left(u_{p}, v_{q}\right)-\Omega_{q}\left(u_{p}, v_{p}\right)\right) \wedge \bar{\alpha}_{p} \\
& +\iint_{M \times M} \chi_{p} d_{p} \zeta^{\delta} \wedge\left(\Omega_{p}\left(u_{p}, v_{q}\right)-\Omega_{p}\left(u_{p}, v_{p}\right)\right) \wedge \bar{\alpha}_{q} \\
= & : I_{2,1}+I_{2,2}+I_{2,3},
\end{aligned}
$$

with obvious notations, where we have used

$$
\begin{aligned}
& \iint_{M \times M} \chi_{p} d_{p} \zeta^{\delta} \wedge \Omega_{p}\left(u_{p}, v_{p}\right) \wedge \bar{\alpha}_{q} \\
& =\int_{M} \chi_{p} \Omega_{p}\left(u_{p}, v_{p}\right) \wedge d_{p}\left(\int_{M} \zeta_{q}^{\delta} \bar{\alpha}_{q}\right)=0
\end{aligned}
$$


by Condition (1) in Definition 2.3. We now analyze the terms in $I_{2}$. For the first term, note that

$$
\begin{aligned}
I_{2,1} & =\int_{M} \chi_{p} \bar{\alpha}_{p}\left(\int_{E_{p}^{\delta}} d_{q} \zeta^{\delta} \wedge \Omega_{q}\left(u_{p}, v_{p}\right)\right) \\
& =-\int_{M} \chi_{p} \bar{\alpha}_{p}\left(\int_{E_{p}^{\delta}} \zeta^{\delta} d \Omega_{q}\left(u_{p}, v_{p}\right)\right) .
\end{aligned}
$$

This integration by parts is possible since $\Omega_{q}\left(u_{p}, v_{p}\right)$ depends on $p$ only through $u$ and $v$, i.e., the explicit spatial dependence is on $q$.

Next, we have

$$
I_{2,2}=\int_{0}^{1} \iint_{M \times M} \chi_{p}\left(v_{q}-v_{p}\right) d_{q} \zeta^{\delta} \wedge \partial_{v} \Omega_{q}\left(u_{p}, v^{*}\right) \wedge \bar{\alpha}_{p} d s,
$$

with $v^{*}=s v_{q}+(1-s) v_{p}$, and a similar expression for $I_{2,3}$. This gives

$$
\begin{aligned}
I_{2,2}+I_{2,3}= & \int_{0}^{1} \iint_{M \times M} \chi\left(v_{q}-v_{p}\right)\left(d_{q} \zeta^{\delta} \wedge \partial_{v} \Omega_{q}\left(u_{p}, v^{*}\right) \wedge \bar{\alpha}_{p}\right. \\
& \left.+d_{p} \zeta^{\delta} \wedge \partial_{v} \Omega_{p}\left(u_{p}, v^{*}\right) \wedge \bar{\alpha}_{q}\right) d s,
\end{aligned}
$$

which, according to Condition (4) of Definition 2.3 and the definition of $\Omega$, yields, for some constant $A>0$,

$$
I_{2,2}+I_{2,3} \leq \int_{M} \oint_{E_{p}^{\delta}} A \chi_{p}\left|v_{q}-v_{p}\right| \bar{\alpha}_{p} \wedge \bar{\alpha}_{q}
$$

Putting these estimates together, we obtain

$$
\begin{aligned}
I_{2} \leq & -\int_{M} \chi_{p} \bar{\alpha}_{p}\left(\int_{E_{p}^{\delta}} \zeta^{\delta} d \Omega_{q}\left(u_{p}, v_{p}\right)\right) \\
& +\int_{M} \oint_{E_{p}^{\delta}} A \chi_{p}\left|v_{q}-v_{p}\right| \bar{\alpha}_{p} \wedge \bar{\alpha}_{q} .
\end{aligned}
$$

Now, we estimate the term $I_{3}$ in (2.19). First, note that

$$
\begin{aligned}
& G_{p}\left(u_{p}, v_{q}\right) \wedge \bar{\alpha}_{q}+G_{q}\left(v_{q}, u_{p}\right) \wedge \bar{\alpha}_{p} \\
& =\operatorname{sgn}\left(u_{p}-v_{q}\right)\left(d \omega_{p}\left(v_{q}\right) \wedge \bar{\alpha}_{q}-d \omega_{q}\left(u_{p}\right) \wedge \bar{\alpha}_{p}\right),
\end{aligned}
$$

and thus

$$
\begin{aligned}
& d \omega_{p}\left(v_{q}\right) \wedge \bar{\alpha}_{q}-d \omega_{q}\left(u_{p}\right) \wedge \bar{\alpha}_{p} \\
& =d \omega_{p}\left(v_{q}\right) \wedge \bar{\alpha}_{q}-d \omega_{q}\left(v_{q}\right) \wedge \bar{\alpha}_{p} \\
& \quad+d \omega_{q}\left(v_{q}\right) \wedge \bar{\alpha}_{p}-d \omega_{q}\left(u_{p}\right) \wedge \bar{\alpha}_{p}=: A_{1}+A_{2} .
\end{aligned}
$$

From Condition (2), that is, $\zeta^{\delta} \leq\left|E_{p}^{\delta}\right|^{-1}$ we find immediately

$$
\int_{M} \chi_{p} \int_{E_{p}^{\delta}} \zeta^{\delta} \operatorname{sgn}\left(u_{p}-v_{q}\right) A_{1} \leq \bar{R}^{\delta}[\omega],
$$

where $\bar{R}^{\delta}[\omega]$ is defined as $R^{\delta}[\omega]$ in the statement of the theorem, but with $\chi_{p} /\left|E_{p}^{\delta}\right|$ instead of $1 /\left|E_{p}^{\delta}\right|$. On the other hand, the term $A_{2}$ gives

$$
\int_{M} \chi_{p} \int_{E_{p}^{\delta}} \zeta^{\delta} \operatorname{sgn}\left(u_{p}-v_{q}\right) A_{2}=-\int_{M} \chi_{p} \bar{\alpha}_{p} \int_{E_{p}^{\delta}} \zeta^{\delta} d \Omega_{q}\left(u_{p}, v_{q}\right) .
$$

This leaves us with

$$
-I_{3} \leq \int_{M} \chi_{p} \bar{\alpha}_{p} \int_{E_{p}^{\delta}} \zeta^{\delta} d \Omega_{q}\left(u_{p}, v_{q}\right)+\bar{R}^{\delta}[\omega]
$$


From the last inequality and (2.20), we obtain

$$
\begin{aligned}
I_{2}-I_{3} & \leq \int_{M} \chi_{p} \bar{\alpha}_{p} \int_{E_{p}^{\delta}} \zeta^{\delta}\left(d \Omega_{q}\left(u_{p}, v_{q}\right)-d \Omega_{q}\left(u_{p}, v_{p}\right)\right) \\
& +\int_{M} \oint_{E_{p}^{\delta}} A \chi_{p}\left|v_{q}-v_{p}\right| \bar{\alpha}_{p} \wedge \bar{\alpha}_{q}+\bar{R}^{\delta}[\omega] .
\end{aligned}
$$

The first integral is bounded by

$$
\int_{M} \chi_{p} \bar{\alpha}_{p} \oint_{E_{p}^{\delta}} \sup _{u}\left|\partial_{u} d \omega_{q}(u) \| v_{p}-v_{q}\right|
$$

so that we finally find

$$
\begin{aligned}
& I_{2}-I_{3} \\
& \leq \int_{M} A \bar{\alpha}_{p} \chi_{p} \oint_{E_{p}^{\delta}}\left(\bar{\alpha}_{q}+\sup _{u}\left|\partial_{u} d \omega_{q}(u)\right|\right)\left|v_{p}-v_{q}\right| \\
& \quad+\bar{R}^{\delta}[\omega] .
\end{aligned}
$$

\section{The terms $I_{1}$ and $I_{4}$.}

Let us now turn to $I_{1}$, the main term in (2.19). As in [4], we choose the test-functions $\chi=\chi^{\epsilon}$ to be supported on $\bigcup_{0 \leq t \leq T+\epsilon} \mathcal{H}_{t}$ and constant within any hypersurface $\mathcal{H}_{t}$, so that $\chi^{\epsilon}$ is a function of $t$ only. Moreover, we arrange that $\chi^{\epsilon} \leq 1, \chi^{\epsilon} \equiv 1$ on $\cup_{\epsilon \leq t \leq T} \mathcal{H}_{t}$, and that $\partial_{t} \chi^{\epsilon}(t) \rightarrow \delta_{t=0}-\delta_{t=T}$ as $\epsilon \rightarrow 0$, where $\delta_{t=\tau}$ is a Dirac mass centered at $\tau$. Also, we have $d \chi^{\epsilon}=\partial_{t} \chi^{\epsilon} d t$.

We now find

$$
\begin{aligned}
I_{1}= & -\iint_{M \times M} \zeta^{\delta} d \chi^{\epsilon} \wedge \Omega_{p}\left(u_{p}, v_{q}\right) \wedge \bar{\alpha}_{q} \\
= & -\iint_{M \times M} \zeta^{\delta} d \chi^{\epsilon} \wedge \Omega_{p}\left(u_{p}, v_{p}\right) \wedge \bar{\alpha}_{q} \\
& +\iint_{M \times M} \zeta^{\delta} d \chi^{\epsilon} \wedge\left(\Omega_{p}\left(u_{p}, v_{p}\right)-\Omega_{p}\left(u_{p}, v_{q}\right)\right) \wedge \bar{\alpha}_{q} .
\end{aligned}
$$

Consider the last integral, and observe that from the non-degeneracy condition (2.5) we find, for any positive real function $g$ supported in $M_{T}$,

$$
\begin{aligned}
& \int_{M} g_{p}\left|d t \wedge \partial_{u} \Omega\right| \leq 2 \sup _{u} \int_{0}^{T} \int_{\mathcal{H}_{t}} g_{p} i^{*} \partial_{u} \omega(u) d t \\
& \leq 2 \bar{c} \int_{M} g_{p} d t \wedge \partial_{u} \omega(0)=2 \bar{c} \int_{M} g_{p} \bar{\alpha}_{p} .
\end{aligned}
$$

Thus, using Condition (2),

$$
\begin{gathered}
\iint_{M \times M} \zeta^{\delta} d \chi^{\epsilon} \wedge\left(\Omega_{p}\left(u_{p}, v_{p}\right)-\Omega_{p}\left(u_{p}, v_{q}\right)\right) \wedge \bar{\alpha}_{q} \\
\quad \leq \int_{M}\left|d \chi^{\epsilon} \wedge \sup _{v} \partial_{v} \Omega_{p}\right| \oint_{E_{p}^{\delta}}\left|v_{p}-v_{q}\right| \bar{\alpha}_{q} \\
\quad \leq \int_{M} 2\left|\partial_{t} \chi^{\epsilon}\right| \bar{c} d t \wedge \partial_{u} \omega_{p}(0) \oint_{E_{p}^{\delta}}\left|v_{p}-v_{q}\right| \bar{\alpha}_{q} \\
\quad=\int_{M} 2\left|\partial_{t} \chi^{\epsilon}\right| \bar{c} \bar{\alpha}_{p} \oint_{E_{p}^{\delta}}\left|v_{p}-v_{q}\right| \bar{\alpha}_{q} .
\end{gathered}
$$


Furthermore, we have from Condition (1) in Definition 2.3,

$$
-\iint_{M \times M} \zeta^{\delta} d \chi^{\epsilon} \wedge \Omega_{p}\left(u_{p}, v_{p}\right) \wedge \bar{\alpha}_{q}=-\int_{M} d \chi^{\epsilon} \wedge \Omega_{p}\left(u_{p}, v_{p}\right) .
$$

Therefore, recalling (2.19) and (2.21), we find

$$
\begin{aligned}
& -\int_{M} d \chi^{\epsilon} \wedge \Omega_{p}\left(u_{p}, v_{p}\right) \\
& \leq \int_{M} 2\left|\partial_{t} \chi^{\epsilon}\right| \bar{c} \bar{\alpha}_{p} \oint_{E_{p}^{\delta}}\left|v_{p}-v_{q}\right| \bar{\alpha}_{q} \\
& \quad+\int_{M} A \bar{\alpha}_{p} \chi^{\epsilon} \oint_{E_{p}^{\delta}}\left(\bar{\alpha}_{q}+\sup _{u}\left|\partial_{u} d \omega_{q}\right|\right)\left|v_{p}-v_{q}\right| \\
& \quad+\bar{R}^{\delta}[\omega]+I_{4} .
\end{aligned}
$$

Let us now take $\epsilon \rightarrow 0$. First, we have

$$
\begin{aligned}
-\underset{\epsilon \rightarrow 0}{\limsup } \int_{M} d \chi^{\epsilon} \wedge \Omega_{p}\left(u_{p}, v_{p}\right) \\
\quad=-\limsup _{\epsilon \rightarrow 0} \int_{0}^{T+\epsilon} \partial_{t} \chi^{\epsilon}(t) \int_{\mathcal{H}_{t}} i^{*} \Omega_{p}\left(u_{p}, v_{p}\right) d t \\
\quad \leq \int_{\mathcal{H}_{T}} i^{*} \Omega_{p}\left(u_{p}, v_{p}\right)-\int_{\mathcal{H}_{0}} i^{*} \Omega_{p}\left(u_{p}, v_{p}\right) .
\end{aligned}
$$

Similarly, and since $\left|\partial_{t} \chi^{\epsilon}(t)\right| \rightarrow \delta_{t=0}+\delta_{t=T}$ as $\epsilon \rightarrow 0$, we find

$$
\begin{aligned}
& \limsup _{\epsilon \rightarrow 0} \int_{M} 2\left|\partial_{t} \chi^{\epsilon}\right| \bar{c} \bar{\alpha}_{p} \oint_{E_{p}^{\delta}}\left|v_{p}-v_{q}\right| \bar{\alpha}_{q} \\
& \quad+\int_{M} A \bar{\alpha}_{p} \chi^{\epsilon} \oint_{E_{p}^{\delta}}\left(\bar{\alpha}_{q}+\sup _{u}\left|\partial_{u} d \omega_{q}\right|\right)\left|v_{p}-v_{q}\right| \\
& \leq R^{\delta}[v]
\end{aligned}
$$

with $R^{\delta}[v]$ defined as in the statement of the theorem. Also, it is clear that $\lim \sup _{\epsilon \rightarrow 0} \bar{R}^{\delta}[\omega] \leq$ $R^{\delta}[\omega]$.

Finally, we must deal with the term

$$
I_{4}=\iint_{M \times M}\left|\alpha_{H} \wedge d_{p}\left(\chi^{\epsilon} \zeta^{\delta}\right)\right| \wedge \bar{\alpha}_{q}+\alpha_{K} \wedge \bar{\alpha}_{q} \chi^{\epsilon} \zeta^{\delta}
$$

We have, using Condition (3) in Definition 2.3 and (2.10),

$$
\begin{aligned}
I_{4} & \leq \int_{M}\left|d \chi^{\epsilon} \wedge \alpha_{H}\right| \int_{M} \zeta^{\delta} \bar{\alpha}_{q}+\frac{1}{\delta} \int_{M} \chi^{\epsilon}\left|\beta_{p} \wedge \alpha_{H}\right| \oint_{E_{p}^{\delta}} \bar{\alpha}_{q} \\
& +\int_{M} \chi^{\epsilon} \alpha_{K} \int_{M} \zeta^{\delta} \bar{\alpha}_{q} .
\end{aligned}
$$

Using Condition (1) and taking the lim sup as $\epsilon \rightarrow 0$ gives $I_{4} \leq R^{\delta}[\alpha]$. This completes the proof of Theorem 2.4. 


\section{Error estimates for a Lorentzian manifold}

\subsection{Conservation laws based on vector fields}

In this section, we derive error estimates for conservation laws posed on Lorentzian manifolds. This is motivated by the fact that Theorem 2.4 is greatly improved if the manifold under consideration has a metric defined on it. To begin with, one does not need to assume the existence of a special family of mollifiers, since these are naturally provided by the metric. Second, one can introduce second order error terms which allow us to consider more general approximate solutions to conservation laws; namely, we obtain an error estimate for very general nonlinear diffusion models; see Theorem 3.5 below.

Let $(M, g)$ be a time-oriented, $(N+1)$-dimensional Lorentzian manifold. Here, $g$ is a metric with signature $(-,+, \ldots,+)$, and we recall that tangent vectors $X \in T_{p} M$ at a point $p \in M$ can be separated into timelike vectors $(g(X, X)<0)$, null vectors $(g(X, X)=0)$, and spacelike vectors $(g(X, X)>0)$. The manifold is assumed to be time-oriented, so that we can distinguish between past-oriented and future-oriented vectors. The Levi-Civita connection associated to $g$ is denoted by $\nabla$ and, for instance, allows us to define the divergence operator $\operatorname{div}_{g}$. Finally, we denote by $d V_{g}$ (or $d V_{g}(p)$, to stress the integration variable) the volume measure determined by the metric $g$.

Following [3], a flux-vector on a manifold is defined as a vector field $f=f_{p}(\bar{u})$ depending on a real parameter $\bar{u}$ and the conservation law on $(M, g)$ associated with $f$ reads

$$
\operatorname{div}_{g}\left(f_{p}(u)\right)=0, \quad u: M \rightarrow \mathbb{R}
$$

Moreover, the flux-vector $f$ is said to be geometry compatible if

$$
\operatorname{div}_{g} f_{p}(\bar{u})=0, \quad \bar{u} \in \mathbb{R}, p \in M,
$$

and to be timelike if its $u$-derivative is a timelike vector field

$$
g\left(\partial_{u} f_{p}(\bar{u}), \partial_{u} f_{p}(\bar{u})\right)<0, \quad p \in M, \bar{u} \in \mathbb{R} .
$$

We are interested in the initial-value problem associated with (3.1). So, we fix a spacelike hypersurface $\mathcal{H}_{0} \subset M$ and a measurable and bounded function $u_{0}$ defined on $\mathcal{H}_{0}$. Then, we search for a function $u=u_{p} \in L^{\infty}(M)$ satisfying (3.1) in the distributional sense and such that the trace of $u$ on $\mathcal{H}_{0}$ coincides with $u_{0}$, that is

$$
u_{\mid \mathcal{H}_{0}}=u_{0} .
$$

It is natural to require that the vectors $\partial_{u} f_{p}(\bar{u})$, which determine the propagation of waves in solutions of (3.1), are timelike and future-oriented. Thus, we will assume throughout that the flux-vector in equation (3.1) is timelike, in the sense of (3.3).

As in the previous section, we assume that the manifold $M$ is globally hyperbolic, which in this Lorentzian setting means that there exists a foliation of $M$ by spacelike, compact, oriented Riemannian hypersurfaces $\mathcal{H}_{t}(t \in \mathbb{R})$ :

$$
M=\bigcup_{t \in \mathbb{R}} \mathcal{H}_{t} .
$$

Any hypersurface $\mathcal{H}_{t_{0}}$ is referred to as a Cauchy surface in $M$, while the family $\mathcal{H}_{t}(t \in \mathbb{R})$ is called an admissible foliation associated with $\mathcal{H}_{t_{0}}$. The future of the given hypersurface will be denoted by

$$
M_{+}:=\bigcup_{t \geq 0} \mathcal{H}_{t} .
$$

Moreover, we denote by $n^{t}$ the future-oriented, normal vector field to each $\mathcal{H}_{t}$, and by $g^{t}$ the induced metric. Finally, along $\mathcal{H}_{t}$, we denote by $X^{t}$ the normal component of a vector field $X$, thus $X^{t}:=g\left(X, n^{t}\right)$. In the following, when there is no risk of confusion, we write $F(u)$ instead of $F_{p}(u)$. 
Definition 3.1. A flux $F=F_{p}(\bar{u})$ is called a convex entropy flux associated with the conservation law (3.1) if there exists a convex function $U: \mathbb{R} \rightarrow \mathbb{R}$ such that

$$
F_{p}(\bar{u})=\int_{0}^{\bar{u}} \partial_{u} U\left(u^{\prime}\right) \partial_{u} f_{p}\left(u^{\prime}\right) d u^{\prime}, \quad p \in M, \bar{u} \in \mathbb{R} .
$$

A measurable and bounded function $u=u_{p}$ is called an entropy solution of the conservation law (3.1)(3.2) if the following entropy inequality

$$
\begin{aligned}
& \int_{M_{+}} g\left(F(u), \nabla_{g} \phi\right) d V_{g}+\int_{M_{+}}\left(\operatorname{div}_{g} F\right)(u) \phi d V_{g} \\
& +\int_{\mathcal{H}_{0}} g_{0}\left(F\left(u_{0}\right), n^{0}\right) \phi_{\mathcal{H}_{0}} d V_{g_{0}}-\int_{M_{+}} U^{\prime}(u)\left(\operatorname{div}_{g} f\right)(u) \phi d V_{g} \geq 0
\end{aligned}
$$

holds for all convex entropy flux $F=F_{p}(\bar{u})$ and all smooth functions $\phi \geq 0$ compactly supported in $M_{+}$.

In particular, the requirements in the above definition imply the inequality

$$
\operatorname{div}_{g}(F(u))-\left(\operatorname{div}_{g} F\right)(u)+U^{\prime}(u)\left(\operatorname{div}_{g} f\right)(u) \leq 0
$$

in the distributional sense. For well-posedness results for the initial value problem (3.1)-(3.4), see [3, 11].

\subsection{Statement of the error estimate}

For convenience, we consider a Riemannian metric $\bar{g}$ associated with the Lorentzian metric $g$. We fix the natural one, that is, in local coordinates where the matrix of the metric $g$ is diagonal, we set $\bar{g}_{11}:=-g_{11}$ and $\bar{g}_{i i}:=g_{i i},(i=2, \ldots, N+1)$. For instance, this allows us to consider the distance function $\ell_{\bar{g}}$ associated with $\bar{g}$. In particular, the volume form and divergence operator associated to $g$ or $\bar{g}$ are the same. Also, we write $B_{p}(r)$ for the geodesic ball centered at $p \in M$ with radius $r$, with respect to the metric $\bar{g}$.

Since we shall rely on Kruzkov's family of entropies for the statement of our results as well as the proofs, we write the conservation law (3.5) with Kruzkov's entropy flux

$$
F_{p}(u, k)=\operatorname{sgn}(u-k)\left(f_{p}(u)-f_{p}(k)\right), \quad k \in \mathbb{R} .
$$

Thus, we are given a bounded measurable function $u$ satisfying an approximate entropy inequality,

$$
\begin{aligned}
\operatorname{div}_{g} & \left(\operatorname{sgn}\left(u_{p}-k\right)\left(f_{p}\left(u_{p}\right)-f_{p}(k)\right)\right)+\operatorname{sgn}\left(u_{p}-k\right) \operatorname{div}_{g} f_{p}(k) \\
\leq & \operatorname{div}_{g} H_{k}+K_{k}+\operatorname{div}_{g_{t}}\left(a_{k} \nabla_{g_{t}} L_{k}\right) .
\end{aligned}
$$

Here, the error terms $H_{k}, K_{k}, L_{k}$ are defined as follows: for each $k \in \mathbb{R}, H_{k}$ is a distributional vector field, that is, an element of the space of linear functionals from the space of smooth 1-forms and taking values in the space of (scalar) distributions on $M$. Thus, for each $\gamma \in \Lambda^{1}(M),\left\langle H_{k}, \gamma\right\rangle \equiv \gamma\left(H_{k}\right)$ is a distribution on $M$, which we assume to be a Radon measure.

The terms $K_{k}$ and $L_{k}$ are Radon measures, and $a_{k}$ are continuously differentiable functions defined on $M$. We suppose that $H_{k}, K_{k}$, and $L_{k}$ satisfy the following uniform bounds (with respect to $k$ ),

$$
\left|\gamma\left(H_{k}\right)\right| \leq \alpha_{H}\left|\gamma^{\sharp}\right|, \quad\left|K_{k}\right| \leq \alpha_{K}, \quad\left|L_{k}\right| \leq \alpha_{L},
$$

for some positive Radon measures $\alpha_{H}, \alpha_{K}, \alpha_{L}$ on $M$. Here, if $\alpha$ is a measure, $|\alpha|$ denotes its variation in the measure-theoretic sense, and $\gamma^{\sharp}$ is the vector obtained from the 1-form $\gamma$ by raising indices using the metric. We also assume that the functions $a_{k}$ satisfy, for some $\alpha_{a}$ independent of $k$,

$$
\left|a_{k}\right|,\left|\nabla_{g^{t}} a_{k}\right|_{g^{t}} \leq \alpha_{a} .
$$


Note that due to the presence of a volume form, a measure on $M$ may be seen indifferently as an element of the dual of the space of $(N+1)$-forms or of the dual of the space of test-functions on $M$. Thus, when we write, for instance, $\int_{M} \alpha_{K} \varphi d V_{\bar{g}}$, this denotes the duality between the (scalar) distribution $\alpha_{K}$ and the $(N+1)$-form $\varphi d V_{\bar{g}}$, and so no regularity is assumed on $\alpha_{K}$.

Now, let $v$ denote the exact solution to the conservation law (3.5), i.e. for all $l \in \mathbb{R}$

$$
\operatorname{div}_{g}\left(\operatorname{sgn}\left(v_{q}-l\right)\left(f_{q}\left(v_{q}\right)-f_{q}(l)\right)\right)+\operatorname{sgn}\left(v_{q}-l\right) \operatorname{div}_{g} f_{q}(l) \leq 0 .
$$

Defining

$$
G_{p}(u, k)=\operatorname{sgn}(u-k) \operatorname{div}_{g} f_{p}(k),
$$

the entropy inequalities read

$$
\operatorname{div}_{g}\left(F_{p}\left(u_{p}, k\right)\right)+G_{p}\left(u_{p}, k\right) \leq \operatorname{div}_{g} H_{k}+K_{k}+\operatorname{div}_{g_{t}}\left(a_{k} \nabla_{g_{t}} L_{k}\right)
$$

for the approximate solution $u$, and

$$
\operatorname{div}_{g}\left(F_{q}\left(v_{q}, l\right)\right)+G_{q}\left(v_{q}, l\right) \leq 0
$$

for the exact solution $v$.

Our main result in this section is Theorem 3.2. It gives a precise, quantitative estimate of the evolution of

$$
\int_{\mathcal{H}_{t}} F^{t}\left(u_{p}, v_{p}\right) d V_{g^{t}}
$$

Note that this quantity is equal to

$$
\int_{\mathcal{H}_{t}}\left|u_{p}-v_{p}\right| d V_{g^{t}}
$$

whenever the flux function $f$ of the equation is such that $f^{t}(u)=u$ for all $u$. Therefore our estimates have the same form as the usual estimates in [4], where the manifold is flat and the time evolution trivial. In the general case, we have $F^{t}(u, v)=\left|f^{t}(u)-f^{t}(v)\right|$ for every $u, v \in \mathbb{R}$. Since $f^{t}$ is, by assumption, a strictly monotone function of $u$, this quantity provides an equivalent measure of the difference between $u$ and $v$ in the $L^{1}$-norm, which takes into account the geometry of the manifold and the structure of the time-evolution of the foliation under consideration.

Theorem 3.2 (Error estimate for conservation laws on a Lorentzian manifold). Let $u$ be a function satisfying the approximate entropy inequalities (3.7), (3.8), and let $v$ be an exact solution satisfying (3.9). Suppose also that $u, v$ are right-continuous with values in $L^{1}\left(\mathcal{H}_{t}\right)$ and that, for some $T \geq 0, \alpha_{H}$ is right-continuous from $[0, T)$ with values in $L^{1}\left(\mathcal{H}_{t}\right)$. For $u \in \mathbb{R}$ and $p \in M$, define the constants

$$
\begin{array}{ll}
\Lambda_{0}:=\sup _{p} \operatorname{Lip}_{u} f^{t}, & \Lambda_{1}:=\sup _{p} \sup _{\substack{X \in T_{p} M \\
|X|=1}} \operatorname{Lip}_{u}\left(\nabla_{X} f\right), \\
\Lambda_{2}:=\sup _{u} \operatorname{Lip}_{p}\left(\operatorname{div}_{g} f\right), & \Lambda_{3}:=\sup _{p} \operatorname{Lip}_{u}\left(\operatorname{div}_{g} f\right) .
\end{array}
$$

Then, for all $\delta>0$ the estimate

$$
\begin{aligned}
& \int_{\mathcal{H}_{T}} F^{t}\left(u_{p}, v_{p}\right) d V_{g^{T}} \\
& \leq \int_{\mathcal{H}_{0}} F^{t}\left(u_{p}, v_{p}\right) d V_{g^{0}}+C\left(E_{v}^{\delta}+E_{f}^{\delta}+E_{H}^{\delta}+E_{K}^{\delta}+E_{L}^{\delta}\right)
\end{aligned}
$$

holds, where $C$ is a constant (which may depend on $N$ ) and

$$
E_{v}^{\delta}:=\left(T \Lambda_{1}+T \Lambda_{3}+\Lambda_{0}\right) \sup _{t \in(0, T)} \int_{\mathcal{H}_{t}} \oint_{B_{p}(\delta)}\left|v_{p}-v_{q}\right| d V_{g} d V_{g^{t}}
$$




$$
\begin{gathered}
E_{f}^{\delta}:=T \sup _{t \in(0, T)}\left|\mathcal{H}_{t}\right| \delta \Lambda_{2}, \quad E_{H}^{\delta}:=\int_{\mathcal{H}_{0} \cup \mathcal{H}_{T}} \alpha_{H} d V_{g^{t}}+\frac{1}{\delta} \int_{0}^{T} \int_{\mathcal{H}_{t}} \alpha_{H} d V_{g^{t}} d t, \\
E_{K}^{\delta}:=\int_{0}^{T} \int_{\mathcal{H}_{t}} \alpha_{K} d V_{g^{t}} d t, \quad E_{L}^{\delta}:=\frac{1}{\delta^{2}} \int_{0}^{T} \int_{\mathcal{H}_{t}} \alpha_{L} \alpha_{a} d V_{g^{t}} d t .
\end{gathered}
$$

\subsection{Application (II). The semi-group contraction property}

Our main result implies a key property of the semi-group of entropy solutions.

Corollary 3.3. In addition to the assumptions of Theorem 3.2. suppose also that the flux $f$ is geometrycompatible and that $v$ has bounded total variation on each slice $\mathcal{H}_{t}$. Then, for every $\delta>0$, the estimate

$$
\begin{aligned}
& \int_{\mathcal{H}_{T}} F^{t}\left(u_{p}, v_{p}\right) d V_{g^{T}} \\
& \leq \int_{\mathcal{H}_{0}} F^{t}\left(u_{p}, v_{p}\right) d V_{g^{0}}+C T \delta \sup _{t} \operatorname{TV}\left(v_{\mid \mathcal{H}_{t}}\right)+C\left(E_{H}^{\delta}+E_{K}^{\delta}+E_{L}^{\delta}\right)
\end{aligned}
$$

holds where $C$ is independent of $\delta$, but depends on $f$, and the error terms are defined as in Theorem 3.2 In particular, if $u$ is also an exact solution, the function

$$
t \mapsto \int_{\mathcal{H}_{t}} F^{t}\left(u_{p}, v_{p}\right) d V_{g^{t}}
$$

is non-increasing.

This result is an immediate consequence of Theorem 3.2 and of the following result, which provides the link between the term $E_{v}^{\delta}$ (associated with the regularity of the exact solution $v$ ) and its total variation. Recall that for each $p \in M$, the exponential map $\exp _{p}: B_{0}(\delta) \rightarrow B_{p}(\delta) \subset M$ provides a diffeomorphism between the ball of radius $\delta$ on the tangent space at $p, B_{0}(\delta) \subset T_{p} M$, and the geodesic ball (according to the Riemannian metric $\bar{g}$ ) $B_{p}(\delta)$ around $p$. (Here, $\delta$ must be small enough.) The exponential map provides a local chart by identifying $T_{p} M$ with $\mathbb{R}^{N+1}$. Also, in what follows, we write $d p, d q$ instead of the volume element on $M$ to keep the exposition uncluttered and to stress the integration variable. It is also more convenient to write $v(p)$ instead of $v_{p}$.

Lemma 3.4. Let $v \in B V\left(\mathcal{H}_{t}\right), t \geq 0$ be a solution to the conservation law (3.9). For $\delta$ sufficiently small, one has

$$
\begin{aligned}
& \int_{\mathcal{H}_{t}} \oint_{B_{p}(\delta)}|v(p)-v(q)| d q d p \\
& \leq C \delta\left(\left(1+\frac{\left\|\operatorname{Lip}_{u} f\right\|_{L^{\infty}(M)}}{\beta}\right) \operatorname{TV}\left(v_{\mid \mathcal{H}_{t}}\right)+\frac{1}{\beta}\left\|\left(\operatorname{div}_{g} f\right)_{\mid \mathcal{H}_{t}}\right\|_{L^{1}\left(\mathcal{H}_{t}\right)}\right),
\end{aligned}
$$

where $\beta=\inf _{u, p} \partial_{u} f^{t}(u, p)>0$. In particular, if the flux $f$ is geometry-compatible, then

$$
\int_{\mathcal{H}_{t}} \oint_{B_{p}(\delta)}|v(p)-v(q)| d q d p \leq C \delta\left(1+\frac{\left\|\operatorname{Lip}_{u} f\right\|_{L^{\infty}(M)}}{\beta}\right) \operatorname{TV}\left(v_{\mid \mathcal{H}_{t}}\right)
$$

Proof. We may assume that the function $v$ is sufficiently smooth since, by a standard density argument, the general result then follows for all functions $v$ with bounded variation. Using the exponential map, we write

$$
\int_{\mathcal{H}_{t}} \oint_{B_{p}(\delta)}|v(p)-v(q)| d q d p=\int_{\mathcal{H}_{t}} \oint_{B_{0}(\delta)}\left|v\left(\exp _{p}(0)\right)-v\left(\exp _{p}(h)\right)\right| d h d p .
$$


Now, consider a partition of unity $\psi_{i}, 1 \leq i \leq m$ subordinate to a covering $\tilde{U}_{i}$ containing a neighborhood of $\mathcal{H}_{t}$ of radius $\delta$, and write $U_{i}=\mathcal{H}_{t} \cap \tilde{U}_{i}$. We have

$$
\begin{aligned}
& \int_{\mathcal{H}_{t}} \oint_{B_{0}(\delta)}\left|v\left(\exp _{p}(0)\right)-v\left(\exp _{p}(h)\right)\right| d h d p \\
& =\sum_{i} \int_{U_{i}} \psi_{i} \oint_{B_{0}(\delta)}\left|v\left(\exp _{p}(0)\right)-v\left(\exp _{p}(h)\right)\right| d h d p \\
& \leq \oint_{B_{0}(\delta)} \sum_{i} \int_{U_{i}} \psi_{i}\left|v\left(\exp _{p}(0)\right)-v\left(\exp _{p}(h)\right)\right| d p d h \\
& \leq C(M) \sup _{h \in B_{0}(\delta)} \sum_{i} \int_{U_{i}} \psi_{i}\left|v\left(\exp _{p}(0)\right)-v\left(\exp _{p}(h)\right)\right| d p,
\end{aligned}
$$

where $C(M)$ is a constant depending on the geometry of $M$. Note that it is not trivial to reverse the order of integration above, and it is necessary to use the partition of unity. This is due to the fact that there may be no way to globally specify the isomorphisms between $B_{0}(\delta) \subset \mathbb{R}^{N+1}$ and $B_{0}(\delta) \subset T M$ in a smooth way. Indeed, a sufficient condition to be able to do so is that the tangent bundle of $M$ is trivial (i.e., diffeomorphic to $M \times T M$ ) in a neighborhood of $\mathcal{H}_{t}$, which may not be the case. Also, $\delta$ must be small enough so that for each $p$ the point $\exp _{p}(h)$ is well defined. We have also abused the notation somewhat since " $d h$ " really stands for the determinant of the Jacobian of the exponential map, which, by compactness, may be uniformly bounded as a function of $p$, whence the constant $C(M)$.

Thus, using that $\exp _{p}(0)=p$, we find

$$
\begin{aligned}
\sum_{i} \int_{U_{i}} \psi_{i}\left|v\left(\exp _{p}(0)\right)-v\left(\exp _{p}(h)\right)\right| d p & \leq \sum_{i} \int_{U_{i}} \psi_{i} \int_{0}^{1} \mid \frac{d}{d s} v\left(\exp _{p}(s h) \mid d s d p\right. \\
& \leq \delta \int_{0}^{1} \sum_{i} \int_{U_{i}} \psi_{i}\left|\nabla_{\bar{g}} v\left(\exp _{p}(s h)\right)\right| d p d s \\
& \leq \delta \int_{\mathcal{H}_{t}}\left|\nabla_{\bar{g}} v(p)\right| d p+A,
\end{aligned}
$$

with

$$
A=\delta \int_{0}^{1} \sum_{i} \int_{U_{i}} \psi_{i}\left|\nabla_{\bar{g}} v\left(\exp _{p}(s h)\right)-\nabla_{\bar{g}} v\left(\exp _{p}(0)\right)\right| d p d s .
$$

Next, split the gradient of $v$ into its time and spatial components,

$$
\int_{\mathcal{H}_{t}}\left|\nabla_{\bar{g}} v(p)\right| d p \leq \int_{\mathcal{H}_{t}}\left|\partial_{t} v(p)\right| d p+\int_{\mathcal{H}_{t}}\left|\nabla_{\bar{g}}^{x} v(p)\right| d p .
$$

Now we use the conservation law to estimate the temporal gradient in terms of the spatial gradient. Consider any system of local coordinates on the leaf $\mathcal{H}_{t}$. A simple computation shows that the conservation law reads for smooth solutions

$$
\partial_{u} f_{p}^{t}(v(p)) \partial_{t} v(p)+\left\langle\partial_{u} f_{p}(v(p)), \nabla_{\bar{g}}^{x} v(p)\right\rangle_{\bar{g}}+\operatorname{div}_{g} f_{p}(v(p))=0 .
$$

Thus, after integrating on $\mathcal{H}_{t}$ we find

$$
\begin{aligned}
\int_{\mathcal{H}_{t}}\left|\partial_{t} v\right| d p & \leq \frac{1}{\beta} \int_{\mathcal{H}_{t}} \partial_{u} f^{t}\left|\partial_{t} v\right| d p \\
& \leq \frac{\left\|\operatorname{Lip}_{u} f\right\|_{L^{\infty}(M)}}{\beta} \int_{\mathcal{H}_{t}}\left|\nabla_{\bar{g}}^{x} v\right| d p+\frac{1}{\beta} \int_{\mathcal{H}_{t}}\left|\operatorname{div}_{g} f_{p}(v(p))\right| d p .
\end{aligned}
$$


Finally, since for smooth $v$

$$
\int_{\mathcal{H}_{t}}\left|\nabla_{\bar{g}}^{x} v(p)\right| d p=\operatorname{TV}\left(v_{\mid \mathcal{H}_{t}}\right)
$$

the result will be proved if $\sup _{h \in B_{0}(\delta)} A=\mathcal{O}\left(\delta^{2}\right)$, which is straightforward. This completes the proof of Lemma 3.4 .

\subsection{Application (III). A nonlinear diffusion model}

In this section, we apply our results to a nonlinear diffusion model on a Lorentzian manifold. For simplicity, we will consider the geometry-compatible case, in which the divergence of the flux vanishes. Also, to shorten the presentation, we assume here that $u$ and $v$ are regular enough so that a weak form of the chain rule applies, see [4] where (in the Euclidian case) the required regularity is that $u, v \in B V_{\text {loc }}$, which is also our case. Following [4], let $\phi: \mathbb{R} \rightarrow \mathbb{R}$ be a Lipschitz continuous function. We consider the nonlinear diffusion equation on $M$,

$$
\operatorname{div}_{g}\left(f_{p}(u)\right)=\Delta_{g^{t}} \phi(u),
$$

where $\Delta_{g^{t}}$ denotes the Laplace-Beltrami operator on the leaf $\mathcal{H}_{t}$. One can check that if $\phi$ is non-decreasing, the approximate entropy inequalities are

$$
\operatorname{div}_{g}\left(\operatorname{sgn}\left(u_{p}-k\right)\left(f_{p}\left(u_{p}\right)-f_{p}(k)\right)\right) \leq \Delta_{g^{t}}|\phi(u)-\phi(k)| .
$$

We will obtain, if $u$ and $v$ have bounded total variation, an error estimate in $\sqrt{\operatorname{Lip} \phi}$, which is the usual estimate in $\sqrt{\epsilon}$ when $\phi(u)=\epsilon u$, and also a finer estimate in which only $v$ is required to have bounded total variation, and $u$ is only required to be bounded in $L^{1}$.

Theorem 3.5. Let $T>0$ and assume that $u$ satisfy the approximate entropy inequalities (3.14) with a Lipschitz continuous nonlinear viscosity $\phi$. Let $v$ be an exact solution of the conservation law (3.9).

1. Suppose that $\operatorname{TV}\left(\left.v\right|_{\mathcal{H}_{t}}\right) \leq V, \operatorname{TV}\left(\left.u\right|_{\mathcal{H}_{t}}\right) \leq U$ for all $0 \leq t \leq T$. Then there is a constant $C>0$ such that

$$
\int_{\mathcal{H}_{T}} F^{t}\left(u_{p}, v_{p}\right) d V_{g^{T}} \leq \int_{\mathcal{H}_{0}} F^{t}\left(u_{p}, v_{p}\right) d V_{g^{0}}+C T \sqrt{(\operatorname{Lip} \phi) V U}
$$

2. Suppose that $\operatorname{TV}\left(\left.v\right|_{\mathcal{H}_{t}}\right) \leq V$, and that $\int_{M_{T}}|u| d V_{g} \leq U$. Then,

$$
\int_{\mathcal{H}_{T}} F^{t}\left(u_{p}, v_{p}\right) d V_{g^{T}} \leq \int_{\mathcal{H}_{0}} F^{t}\left(u_{p}, v_{p}\right) d V_{g^{0}}+C T(Q U)^{1 / 3} V^{2 / 3},
$$

with $Q=\sup _{u \neq 0}|\phi(u)-\phi(0)| /|u|$.

Proof. Apply Corollary 3.3 with $H_{k}$ as the sole error term. We find (in the sense of measures)

$$
\left|H_{k}\right| \leq \operatorname{Lip}_{u} \phi\left|\nabla_{g} u\right|=: \alpha_{H} .
$$

Estimating the term $R^{\delta}[v]$ using Lemma 3.4 gives

$$
\begin{aligned}
& \int_{\mathcal{H}_{T}} F^{t}\left(u_{p}, v_{p}\right) d V_{g^{T}} \\
& \leq \int_{\mathcal{H}_{0}} F^{t}\left(u_{p}, v_{p}\right) d V_{g^{0}}+C T \delta V+C \frac{T}{\delta} \operatorname{Lip} \phi U,
\end{aligned}
$$

and the estimate (3.15) follows by minimizing with respect to $\delta$. 
To establish (3.16), write $L_{k}=|\phi(u)-\phi(k)|-|\phi(0)-\phi(k)|$, and so $\left|L_{k}\right| \leq Q|u|=: \alpha_{L}$. Now we apply Corollary 3.3 and Lemma 3.4 to find

$$
\begin{aligned}
& \int_{\mathcal{H}_{T}} F^{t}\left(u_{p}, v_{p}\right) d V_{g^{T}} \\
& \leq \int_{\mathcal{H}_{0}} F^{t}\left(u_{p}, v_{p}\right) d V_{g^{0}}+C T \delta V+C \frac{T}{\delta^{2}} Q U,
\end{aligned}
$$

from which the estimate (3.16) follows by choosing the optimal value of $\delta$. This completes the proof of Theorem 3.5 .

\subsection{Derivation of the error estimate}

We provide here the proof of Theorem 3.2 Let $\varphi$ be a smooth, compactly supported function on $M \times M$. From (3.8), (3.10) and (3.11), we have for all $k, l \in \mathbb{R}$

$$
\begin{aligned}
& -\int_{M} d_{p} \varphi\left(F_{p}\left(u_{p}, k\right)\right) d V_{g}(p)+\int_{M} \varphi_{p, q} G_{p}\left(u_{p}, k\right) d V_{g}(p) \\
& \leq \int_{M}\left(\operatorname{div}_{g} H_{k}+K_{k}+\operatorname{div}_{g^{t}}\left(a_{k} \nabla_{g^{t}} L_{k}\right)\right) \varphi_{p, q} d V_{g}(p) \\
& \leq \int_{M}\left|d \varphi\left(H_{k}\right)\right| d V_{g}(p)+\int_{M}\left|K_{k}\right| \varphi d V_{g}(p)+\int_{M}\left|L_{k}\right| \operatorname{div}_{g^{t}}\left(a_{k} \nabla_{g^{t}} \varphi\right) d V_{g}(p) \\
& \leq \int_{M} E(\varphi) d V_{g}(p),
\end{aligned}
$$

with

$$
E(\varphi):=\alpha_{H}\left|\nabla_{g} \varphi\right|+\alpha_{K} \varphi+\alpha_{L} \alpha_{a}\left(\left|\Delta_{g^{t}} \varphi\right|+\left|\nabla_{g^{t}} \varphi\right|\right),
$$

and similarly

$$
-\int_{M} d_{q} \varphi\left(F_{q}\left(v_{q}, l\right)\right) d V_{g}(q)+\int_{M} \varphi_{p, q} G_{q}\left(v_{q}, l\right) d V_{g}(q) \leq 0,
$$

where we recall that $d_{p}$ (resp., $d_{q}$ ) denotes the differential of a function only with respect to $p$ (resp., $q$ ). Taking $k=v_{q}, l=u_{p}$, integrating over $M$, summing the above inequalities, and using Fubini's theorem, we find

$$
\begin{aligned}
& -\iint_{M \times M}\left\{d_{p} \varphi\left(F_{p}\left(u_{p}, v_{q}\right)\right)+d_{q} \varphi\left(F_{q}\left(u_{p}, v_{q}\right)\right)\right\} d V_{g}(p) d V_{g}(q) \\
& \quad+\iint_{M \times M} \varphi_{p, q}\left(G_{p}\left(u_{p}, v_{q}\right)+G_{q}\left(v_{q}, u_{p}\right)\right) d V_{g}(p) d V_{g}(q) \\
& \leq \iint_{M \times M} E(\varphi) d V_{g}(p) d V_{g}(q) .
\end{aligned}
$$

Note also that

$$
\begin{aligned}
& G_{p}\left(u_{p}, v_{q}\right)+G_{q}\left(v_{q}, u_{p}\right) \\
& =\operatorname{sgn}\left(u_{p}-v_{q}\right)\left(\operatorname{div}_{g} f_{p}\left(v_{q}\right)-\operatorname{div}_{g} f_{q}\left(u_{p}\right)\right) .
\end{aligned}
$$

Now take

$$
\varphi_{p, q}=\chi_{p} \xi_{p, q}^{\delta}
$$

where the function $\xi^{\delta}$ is of the form $\xi_{p, q}^{\delta}=\zeta^{\delta}\left(\ell_{\bar{g}}(p, q)\right)$, the function $\chi$ is to be chosen later, and $\ell_{\bar{g}}$ denotes the distance function associated with the Riemannian metric $\bar{g}$. Also, we take $\zeta^{\delta}$ to be a standard family of mollifiers with respect to the Riemannian metric (for a precise definition, see [10]). Here, we only record the properties which we will need, namely

$$
\int_{M} \xi^{\delta} d V_{\bar{g}}=1, \quad\left|\nabla_{\bar{g}} \xi^{\delta}\right| \leq \frac{C}{\delta^{N+1}}, \quad\left|\Delta_{\bar{g}} \xi^{\delta}\right| \leq \frac{C}{\delta^{N+2}} .
$$


In what follows we omit the superscript $\delta$ when there is no risk of confusion. One can check that the function $\ell_{\bar{g}}$ satisfies

$$
d_{q} \ell_{\bar{g}}(X)=\left\langle K_{q}, X\right\rangle_{\bar{g}}, \quad d_{p} \ell_{\bar{g}}(X)=-\left\langle K_{p}, X\right\rangle_{\bar{g}}
$$

for all vector fields $X$, where $K_{p}$ is the unit tangent vector at $p$ to the (unique) geodesic connecting $p$ and $q$. Therefore, for all vector fields $X$, we have

$$
d_{q} \xi(X)=\zeta^{\prime} \circ \ell_{\bar{g}}\left\langle K_{q}, X\right\rangle_{\bar{g}}, \quad d_{p} \xi(X)=-\zeta^{\prime} \circ \ell_{\bar{g}}\left\langle K_{p}, X\right\rangle_{\bar{g}} .
$$

Also, when there is no risk of confusion, we shall simply write $\zeta^{\prime}$ instead of $\zeta^{\prime} \circ \ell_{\bar{g}}$. We find from (3.18)

$$
\begin{aligned}
& \iint_{M \times M}\left(-\xi d \chi F_{p}(u, v)-\chi\left(d_{p} \xi F_{p}(u, v)+d_{q} \xi F_{q}(u, v)\right)\right. \\
& \left.\quad-\chi \xi \operatorname{sgn}(u-v)\left(\operatorname{div}_{g} f_{q}(u)-\operatorname{div}_{g} f_{p}(v)\right)\right) d V_{g}(p) d V_{g}(q) \\
& \leq \iint_{M \times M} E(\chi \xi) d V_{g}(p) d V_{g}(q),
\end{aligned}
$$

where $u=u_{p}$ and $v=v_{q}$. We now write the last inequality as

$$
I_{1} \leq I_{2}+I_{3}+I_{4}
$$

with

$$
\begin{aligned}
I_{1} & =\iint_{M \times M}-\xi d \chi F_{p}(u, v) d V_{g}(p) d V_{g}(q), \\
I_{2} & =\iint_{M \times M} \chi\left(d_{p} \xi F_{p}(u, v)+d_{q} \xi F_{q}(u, v)\right) d V_{g}(p) d V_{g}(q), \\
I_{3} & =\iint_{M \times M} \chi \xi \operatorname{sgn}(u-v)\left(\operatorname{div}_{g} f_{q}(u)-\operatorname{div}_{g} f_{p}(v)\right) d V_{g}(p) d V_{g}(q), \\
I_{4} & =\iint_{M \times M} E(\chi \xi) d V_{g}(p) d V_{g}(q) .
\end{aligned}
$$

\section{The term $I_{2}$}

Note that the term $I_{2}$ vanishes in the "homogenous" Euclidian case. Writing $I_{2}=\int_{M} \chi_{p} I_{2}^{\prime} d V_{g}(p)$, we can write

$$
\begin{aligned}
I_{2}^{\prime}= & \int_{M} d_{q} \xi\left(F_{q}\left(u_{p}, v_{p}\right)\right) d V_{g}(q)+\int_{M} d_{q} \xi\left(F_{q}\left(u_{p}, v_{q}\right)-F_{q}\left(u_{p}, v_{p}\right)\right) d V_{g}(q) \\
& +\int_{M} d_{p} \xi\left(F_{p}\left(u_{p}, v_{q}\right)-F_{p}\left(u_{p}, v_{p}\right)\right) d V_{g}(q) \\
= & I_{2,1}+I_{2,2}+I_{2,3},
\end{aligned}
$$

since

$$
\int_{M} d_{p} \xi\left(F_{p}\left(u_{p}, v_{p}\right)\right) d V_{g}(q)=0 .
$$

To see this, note that $F_{p}\left(u_{p}, v_{p}\right)$ does not depend on $q$, and thus

$$
F^{i} \int_{M} \partial_{p^{i}} \xi d V(q)=F^{i} \partial_{p^{i}}\left(\int_{M} \xi d V(q)\right)=F^{i} \partial_{p^{i}}(1)=0 .
$$

We have

$$
\int_{M} d_{q} \xi\left(F_{q}\left(u_{p}, v_{p}\right)\right) d V_{g}(q)=-\int_{M} \xi \operatorname{div}_{g} F_{q}\left(u_{p}, v_{p}\right) d V_{g}(q),
$$


since we can integrate by parts in this term, as $v$ and $u$ do not depend on the integration variable $q$. The remaining terms are estimated using the regularity of the flux and the difference $\left|v_{p}-v_{q}\right|$, as follows. From (3.22) we find

$$
\begin{aligned}
& I_{2,2}+I_{2,3} \\
& =\int_{0}^{1} \int_{M}\left(v_{q}-v_{p}\right)\left(d_{q} \xi\left(\partial_{v} F_{q}\left(u_{p}, v^{*}\right)\right)-d_{p} \xi\left(\partial_{v} F_{p}\left(u_{p}, v^{*}\right)\right)\right) d V_{g}(q) d s,
\end{aligned}
$$

with $v^{*}=s v_{q}+(1-s) v_{p}$. From (3.6), (3.20), we have

$$
d_{p} \xi F_{p}(u, v)+d_{q} \xi F_{q}(u, v)=-\zeta^{\prime}\left(\left\langle K_{p}, F_{p}(u, v)\right\rangle_{\bar{g}}+\left\langle K_{q}, F_{q}(u, v)\right\rangle_{\bar{g}}\right) .
$$

Now, consider the parallel transport of vectors along a curve on $M$ as follows. Let $\gamma:\left[0, t_{0}\right] \rightarrow$ $M$ be a smooth curve, and $\nabla$ the covariant derivative operator associated to the Riemannian metric $\bar{g}$. Given $0 \leq s \leq t \leq t_{0}$, the parallel transport is the operator $\tau_{s, t}: T_{\gamma(s)} M \rightarrow T_{\gamma(t)} M$ such that, given a vector $X_{\gamma(s)} \in T_{\gamma(s)} M$, then $\tau_{s, t} X_{\gamma(s)} \in T_{\gamma(t)} M$ is the unique solution of the differential equation

$$
\nabla_{\gamma^{\prime}} X=0,\left.\quad \tau_{s, t} X_{\gamma(s)}\right|_{t=s}=X_{\gamma(s)} .
$$

For our purposes, it is more important to note that, conversely, one may recover the covariant derivative from the notion of parallel transport, using the following relation,

$$
\nabla_{\gamma^{\prime}(0)} X=\lim _{h \rightarrow 0} \frac{\tau_{h, 0} X_{\gamma(h)}-X_{\gamma(0)}}{h} .
$$

Also, the parallel transport enjoys the property of preserving scalar products, that is, for all vector fields $X, Y$ defined along the curve $\gamma$,

$$
\left\langle X_{\gamma(s)}, Y_{\gamma(s)}\right\rangle_{\bar{g}_{\gamma(s)}}=\left\langle\tau_{s, t} X_{\gamma(s)}, \tau_{s, t} Y_{\gamma(s)}\right\rangle_{\bar{g}_{(t)}} .
$$

Furthermore, if $\gamma$ is a geodesic curve, then its tangent vector is invariant under parallel transport. With the notations above, if $p=\gamma(0)$ and $q=\gamma(h)$, then $\tau_{0, h} K_{p}=K_{q}$. Using the above properties of the parallel transport, we find

$$
\begin{aligned}
\left\langle K_{p}, F_{p}(u, v)\right\rangle_{\bar{g}}-\left\langle K_{q}, F_{q}(u, v)\right\rangle_{\bar{g}} & =-h\left\langle K_{p}, \frac{\tau_{h, 0} F_{q}(u, v)-F_{p}(u, v)}{h}\right\rangle_{\bar{g}} \\
& =-h\left\langle K_{p^{*}}, \nabla_{K_{p^{*}}} F_{p^{*}}(u, v)\right\rangle_{\bar{g}}
\end{aligned}
$$

with $h=\ell_{\bar{g}}(p, q)$ and $p^{*}$ some point on the geodesic from $p$ to $q$. We write simply $|X|$ for the norm of a vector with respect to the reference Riemannian metric to keep the notations simple. From (3.22), (3.23) and (3.27), with $\partial_{v} F$ instead of $F$, we deduce

$$
\begin{aligned}
I_{2}^{\prime} \leq & \int_{M} \xi \operatorname{div}_{g} F_{q}\left(u_{p}, v_{p}\right) d V_{g}(q) \\
& +\int_{0}^{1} \int_{M} h\left|v_{p}-v_{q}\right|\left|\left\langle\zeta^{\prime} K_{p^{*}},\left(\nabla_{K_{p^{*}}} \partial_{v} F\right)_{p^{*}}\left(u_{p}, v^{*}\right)\right\rangle_{\bar{g}}\right| d V_{g}(q) d s .
\end{aligned}
$$

Now, since $|K|=1$ and $h \leq \delta$,

$$
\begin{aligned}
& \int_{0}^{1} \int_{M} h\left|v_{p}-v_{q}\right|\left|\left\langle\zeta^{\prime} K_{p^{*}},\left(\nabla_{K_{p^{*}}} \partial_{v} F\right)_{p^{*}}\left(u_{p}, v^{*}\right)\right\rangle_{\bar{g}}\right| d V_{g}(q) d s \\
& \leq \Lambda_{1} \int_{M} \delta\left|\zeta^{\prime} \| v_{p}-v_{q}\right| d V_{g}(q),
\end{aligned}
$$

where $\Lambda_{1}$ is defined in (3.12). Thus

$$
\begin{aligned}
I_{2} \leq & -\iint_{M \times M} \chi \xi \operatorname{div}_{g} F_{q}\left(u_{p}, v_{p}\right) d V_{g}(q) d V_{g}(p) \\
& +\Lambda_{1} \iint_{M \times M} \chi \delta\left|\zeta^{\prime} \| v_{p}-v_{q}\right| d V_{g}(q) d V_{g}(p) .
\end{aligned}
$$




\section{The term $I_{3}$}

We now turn to the term $I_{3}$ in (3.21), and write

$$
\operatorname{div}_{g} f_{p}\left(v_{q}\right)-\operatorname{div}_{g} f_{q}\left(u_{p}\right)=\operatorname{div}_{g} f_{p}\left(v_{q}\right)-\operatorname{div}_{g} f_{q}\left(v_{q}\right)+\operatorname{div}_{g} f_{q}\left(v_{q}\right)-\operatorname{div}_{g} f_{q}\left(u_{p}\right),
$$

thus

$$
\begin{aligned}
I_{3}= & -\iint_{M \times M} \chi \xi \operatorname{sgn}\left(u_{p}-v_{q}\right)\left\{\operatorname{div}_{g} f_{p}\left(v_{q}\right)-\operatorname{div}_{g} f_{q}\left(v_{q}\right)\right\} d V_{g}(q) d V_{g}(p) \\
& -\iint_{M \times M} \chi \xi \operatorname{sgn}\left(u_{p}-v_{q}\right)\left\{\operatorname{div}_{g} f_{q}\left(v_{q}\right)-\operatorname{div}_{g} f_{q}\left(u_{p}\right)\right\} d V_{g}(q) d V_{g}(p) .
\end{aligned}
$$

The first term is bounded by

$$
\iint_{M \times M} \chi \xi \delta \Lambda_{2} d V_{g}(q) d V_{g}(p),
$$

while the second term is simply

$$
\iint_{M \times M} \chi \xi \operatorname{div}_{g} F_{q}\left(u_{p}, v_{q}\right) d V_{g}(q) d V_{g}(p) .
$$

Thanks to (3.28), this leads to

$$
\begin{aligned}
\left|I_{2}+I_{3}\right| \leq & \iint_{M \times M} \chi \xi\left|\operatorname{div}_{g} F_{q}\left(u_{p}, v_{q}\right)-\operatorname{div}_{g} F_{q}\left(u_{p}, v_{p}\right)\right| d V_{g}(q) d V_{g}(p) \\
& +\iint_{M \times M} \Lambda_{1} \chi \delta\left|\zeta^{\prime}\right|\left|v_{p}-v_{q}\right|+\delta \Lambda_{2} \chi \xi d V_{g}(q) d V_{g}(p) .
\end{aligned}
$$

We have

$$
\begin{aligned}
& \iint_{M \times M} \chi \xi\left|\operatorname{div}_{g} F_{q}\left(u_{p}, v_{q}\right)-\operatorname{div}_{g} F_{q}\left(u_{p}, v_{p}\right)\right| d V_{g}(q) d V_{g}(p) \\
& \leq \iint_{M \times M} \chi \xi \operatorname{Lip}_{u}\left(\operatorname{div}_{g} f\right)\left|v_{p}-v_{q}\right| d V_{g}(q) d V_{g}(p)
\end{aligned}
$$

which gives

$$
\left|I_{2}+I_{3}\right| \leq \iint_{M \times M} \chi\left(\Lambda_{1} \delta\left|\zeta^{\prime}\right|+\Lambda_{3} \xi\right)\left|v_{p}-v_{q}\right|+\delta \Lambda_{2} \chi \xi d V_{g}(q) d V_{g}(p) .
$$

\section{The term $I_{1}$}

We now treat the main term $I_{1}$. We take $\chi$ to be a function which is compactly supported in time and constant along the hypersurfaces $\mathcal{H}_{t}$ and, thus, for all tangent vectors $Y$, we have $d \chi(Y)=\partial_{t} \chi Y^{t}$. First, we have

$$
\begin{aligned}
I_{1}= & \iint_{M \times M}-\xi \partial_{t} \chi F_{p}^{t}\left(u_{p}, v_{p}\right) d V_{g}(p) d V_{g}(q) \\
& -\iint_{M \times M} \xi \partial_{t} \chi\left(F_{p}^{t}\left(u_{p}, v_{q}\right)-F_{p}^{t}\left(u_{p}, v_{p}\right)\right) d V_{g}(p) d V_{g}(q) .
\end{aligned}
$$

Now,

$$
\begin{aligned}
& \iint_{M \times M} \xi \partial_{t} \chi\left(F_{p}^{t}\left(u_{p}, v_{q}\right)-F_{p}^{t}\left(u_{p}, v_{p}\right)\right) d V_{g}(p) d V_{g}(q) \\
& \leq \iint_{M \times M} \operatorname{Lip}_{u} f^{t} \xi\left|\partial_{t} \chi \| v_{p}-v_{q}\right| d V_{g}(p) d V_{g}(q),
\end{aligned}
$$


where we have used the fact that, since $\partial_{u} f^{t}>0$, we have $\left|f^{t}(v)-f^{t}(u)\right|=F^{t}(u, v)$. From the last inequality and (3.28), (3.29), the inequality (3.21) becomes

$$
\begin{aligned}
& \iint_{M \times M}-\xi \partial_{t} \chi F_{p}^{t}\left(u_{p}, v_{p}\right) d V_{g}(p) d V_{g}(q) \\
& \leq \iint_{M \times M} \Phi_{1}(p, q)\left|v_{p}-v_{q}\right| d V_{g}(p) d V_{g}(q)+\iint_{M \times M} \delta \chi \xi \Lambda_{2} d V_{g}(p) d V_{g}(q)+I_{4},
\end{aligned}
$$

with

$$
\Phi_{1}=\chi\left(\Lambda_{1} \delta\left|\zeta^{\prime}\right|+\xi \Lambda_{3}\right)+\left|\partial_{t} \chi\right| \xi \Lambda_{0}
$$

and where the constants $\Lambda_{i}$ are given by (3.12). Consider now the first term of (3.30). From the properties of the mollifiers $\xi$, namely $\int_{M} \xi_{p, q} d V_{g}(q)=1$ for all $p \in M$, we find

$$
\iint_{M \times M}-\xi \partial_{t} \chi F_{p}^{t}\left(u_{p}, v_{p}\right) d V_{g}(p) d V_{g}(q)=\int_{M}-\partial_{t} \chi F_{p}^{t}\left(u_{p}, v_{p}\right) d V_{g}(p) .
$$

We choose the function $\chi=\chi^{\epsilon} \in(0,1)$ (which only depends on the $t$ coordinate and the small parameter $\epsilon>0)$, to be identically one if $t \in(\epsilon, T)$, supported on the set

$$
\bigcup_{t \in(0, T+\epsilon)} \mathcal{H}_{t}
$$

so that its derivative, $\partial_{t} \chi$, is supported in

$$
\bigcup_{t \in(0, \epsilon) \cup(T, T+\epsilon)} \mathcal{H}_{t}
$$

and satisfying $\chi^{\epsilon} \rightarrow \mathbb{1}_{\left(\mathcal{H}_{t}\right)_{\epsilon \in(0, T)}}$ as $\epsilon \rightarrow 0$. Therefore, since $\partial_{t} \chi$ approaches $\delta_{\mathcal{H}_{0}}-\delta_{\mathcal{H}_{T}}$, and in view of the regularity assumptions on $u$ and $v$ and of (3.32), we get

$$
\begin{aligned}
& \limsup _{\epsilon \rightarrow 0} \int_{M}-\partial_{t} \chi^{\epsilon} F_{p}^{t}\left(u_{p}, v_{p}\right) d V_{g}(p) \\
& \leq \int_{\mathcal{H}_{T}} F_{p}^{t}\left(u_{p}, v_{p}\right) d V_{g}(p)-\int_{\mathcal{H}_{0}} F_{p}^{t}\left(u_{p}, v_{p}\right) d V_{g}(p) .
\end{aligned}
$$

Next, consider the second term in (3.30). It yields the term $E_{v}^{\delta}$ depending on the regularity of $v$, as follows. In view of (3.31) and since there exists a constant $C_{N}$ such that

$$
\xi \Lambda_{3}+\Lambda_{1} \delta\left|\zeta^{\prime}\right| \leq \frac{C_{N}}{\delta^{N+1}}\left(\Lambda_{1}+\Lambda_{3}\right), \quad \sup _{p \in M} \frac{\left|B_{p}(\delta)\right|}{\delta^{N+1}} \leq C_{N},
$$

we find

$$
\begin{aligned}
& \limsup _{\epsilon \rightarrow 0} \Lambda_{0} \iint_{M \times M} \xi\left|\partial_{t} \chi^{\epsilon}\right|\left|v_{p}-v_{q}\right| d V_{g}(q) d V_{g}(p) \\
& \leq C_{N} \Lambda_{0} \max _{t=0, T} \int_{\mathcal{H}_{t}} \oint_{B_{p}(\delta)}\left|v_{p}-v_{q}\right| d V_{g}(q) d V_{g}(p)
\end{aligned}
$$

and

$$
\begin{aligned}
& \underset{\epsilon \rightarrow 0}{\limsup } \iint_{M \times M} \chi^{\epsilon}\left(\xi \Lambda_{3}+\Lambda_{1} \delta\left|\zeta^{\prime}\right|\right)\left|v_{p}-v_{q}\right| d V_{g}(q) d V_{g}(p) \\
& \leq T C_{N}\left(\Lambda_{1}+\Lambda_{3}\right) \sup _{t \in 0, T} \int_{\mathcal{H}_{t}} \oint_{B_{p}(\delta)}\left|v_{p}-v_{q}\right| d V_{g}(q) d V_{g}(p) .
\end{aligned}
$$


Thus, we have

$$
\begin{aligned}
& \limsup _{\epsilon \rightarrow 0} \iint_{M \times M} \Phi_{1}(p, q)\left|v_{p}-v_{q}\right| d V_{g}(q) d V_{g}(p) \\
& \leq C_{N}\left(T \Lambda_{1}+T \Lambda_{3}+\Lambda_{0}\right) \sup _{t \in 0, T} \int_{\mathcal{H}_{t}} \oint_{B_{p}(\delta)}\left|v_{p}-v_{q}\right| d V_{g}(q) d V_{g}(p) .
\end{aligned}
$$

Now, considering the last term in (3.30), we find

$$
\iint_{M \times M} \delta \Lambda_{2} \chi \xi d V_{g}(q) d V_{g}(p)=\delta \Lambda_{2} \int_{M} \chi d V_{g}(p) \leq T \delta \sup _{t \in(0, T)}\left|\mathcal{H}_{t}\right| \Lambda_{2}
$$

Finally, consider the error term $I_{4}$ in (3.21), given by (3.17). First, note that

$$
d_{p} \varphi=\xi d \chi+\chi d_{p} \xi, \quad \nabla_{g^{t}} \varphi=\chi \nabla_{g^{t}} \xi, \quad \Delta_{g^{t}} \varphi=\chi \Delta_{g^{t}} \xi .
$$

From the properties of the test-functions $\xi$ and $\chi^{\epsilon},(3.19)$, from (3.34), and using the regularity of $\alpha_{H}$, we find

$$
\begin{aligned}
\limsup _{\epsilon \rightarrow 0} \iint_{M \times M}\left|d \chi^{\epsilon}\right| \xi \alpha_{H} d V_{g}(p) d V_{g}(q) & \leq \limsup _{\epsilon \rightarrow 0} \int_{M}\left|\partial_{t} \chi^{\epsilon}\right| \alpha_{H} d V_{g}(p) \\
& \leq \sum_{t=0, T} \int_{\mathcal{H}_{t}} \alpha_{H} d V_{g}(p) \\
& \limsup _{\epsilon \rightarrow 0} \iint_{M \times M}\left|d_{p} \xi\right| \chi^{\epsilon} \alpha_{H} d V_{g}(q) d V_{g}(p) \\
\leq & \limsup _{\epsilon \rightarrow 0} \int_{M} \chi^{\epsilon} \int_{B_{p}(\delta)}\left|\zeta_{p_{,}}^{\prime}\right| \alpha_{H} d V_{g}(q) d V_{g}(p) \\
\leq & \limsup _{\epsilon \rightarrow 0} \int_{M} \chi^{\epsilon} \frac{1}{\delta} \oint_{B_{p}(\delta)} \frac{\left|B_{p}(\delta)\right|}{\delta^{N+1}} d V_{g}(q) d V_{g}(p) \\
\leq & \limsup _{\epsilon \rightarrow 0} \frac{C}{\delta} \int_{M} \chi^{\epsilon} \alpha_{H} d V_{g}(p) \leq \frac{C}{\delta} \int_{M_{T}} \alpha_{H} d V_{g}(p)
\end{aligned}
$$

then

$$
\limsup _{\epsilon \rightarrow 0} \iint_{M \times M} \xi \chi^{\epsilon} \alpha_{K} d V_{g}(p) d V_{g}(q) \leq \int_{M_{T}} \alpha_{K} d V_{g}(p)
$$

and

$$
\begin{aligned}
& \limsup _{\epsilon \rightarrow 0} \iint_{M \times M} \alpha_{L} \alpha_{a}\left(\left|\Delta_{g^{t}} \varphi\right|+\left|\nabla_{g^{t}} \varphi\right|\right) d V_{g}(p) d V_{g}(q) \\
& \leq C\left(\frac{1}{\delta}+\frac{1}{\delta^{2}}\right) \int_{M_{T}} \alpha_{L} \alpha_{a} d V_{g}(p) .
\end{aligned}
$$

The estimate (3.13) now follows from the inequalities above and from (3.33), (3.35), (3.36). This completes the proof of Theorem 3.2

\section{Acknowledgements}

This paper was written when the second author (PLF) participated in the international research program on "Nonlinear Partial Differential Equations", hold at the Centre for Advanced Study, Norwegian Academy of Sciences and Letters during the Academic Year 2008-09. This author is grateful to Helge Holden and Kenneth Karlsen for their invitation and hospitality.

The authors were supported by the Agence Nationale de la Recherche (ANR) via the grant 062-134423. PA was also supported by the Portuguese Foundation for Science and Technology (FCT) 
through the post-doctoral fellowship SFRH/BPD/43548/2008, and by a Ciência 2008 fellowship. WN was also supported by FAPERJ via the grant E-26/111.564/2008 entitled "Analysis, Geometry and Applications", by Pronex-FAPERJ through the grant E-26/ 110.560/2010 entitled "Nonlinear Partial Differential Equations", and by a joint project between Brazil and France.

\section{References}

[1] Amorim P., Ben-Artzi M., And LeFloch P.G., Hyperbolic conservation laws on manifolds. Total variation estimates and the finite volume method, Meth. Appl. Anal. 12 (2005), 291-324.

[2] Амоrim P., LeFloch P.G., and Oкutmustur B., Finite volume schemes on Lorentzian manifolds, Comm. Math. Sc. 6 (2008), 1059-1086.

[3] Ben-Artzi M. And LeFloch P.G., The well-posedness theory for geometry compatible, hyperbolic conservation laws on manifolds, Ann. I. H. Poincare - AN 24 (2007), 989-1008.

[4] Bouchut F. And Perthame, B., Kruzkov's estimates for scalar conservation laws revisited, Trans. Amer. Math. Soc. 350 (1998), 2847-2870.

[5] Cockburn B., Coquel F., and LeFloch P.G., Convergence of finite volume methods for multidimensional conservation laws, SIAM J. Numer. Anal. 32 (1995), 687-705.

[6] KRUzKov S.N., First-order quasilinear equations with several space variables, Math. USSR Sb. 10 (1970), 217-243.

[7] Kuznetsov N.N., Accuracy of some approximate methods for computing the weak solutions of a first-order quasi-linear equations, USSR Comput. Math. Math. Phys. 16 (1976), 105-119.

[8] Kuznetsov N.N., On stable methods for solving nonlinear first-order partial differential equations in the class of discontinuous solutions, Topics Numerical Analysis III (Proc. Roy. Irish Acad. Conf.), Trinity College, Dublin (1976), pp. 183-192.

[9] LeFloch P.G., Hyperbolic conservation laws on spacetime, Summer Program on "Nonlinear Conservation laws and applications", Ed. by A. Bressan, G.-Q. Chen, M. Lewicka, and D. Wang, July 13-31, 2009, IMA publication, Minneapolis, 2010.

[10] LeFloch P.G., Neves, W., and Okutmustur B., Hyperbolic conservation laws on manifolds. Error estimate for finite volume schemes, Acta Math. Sinica 25 (2009), 1041-1066.

[11] LeFloch P.G. and Okutmustur B., Hyperbolic conservation laws on spacetimes. A finite volume scheme based on differential forms, Far East J. Math. Sci. 31 (2008), 49-83.

[12] Panov E.Y., On the Cauchy problem for a first-order quasilinear equation on a manifold, Differential Equations 33 (1997), 257-266.

[13] PAnov E.Y., On the Dirichlet problem for first-order quasilinear equations on a manifold, to appear. 